\title{
Gauduchon metrics with prescribed volume form
}

\author{
by \\ GÁBOR SZÉKELYHIDI \\ University of Notre Dame \\ Notre Dame, IN, U.S.A. \\ VALentino Tosatti \\ Northwestern University \\ Evanston, IL, U.S.A.
}

Ben Weinkove

Northwestern University Evanston, IL, U.S.A.

\section{Introduction}

Let $M$ be a compact complex manifold of complex dimension $n$. Suppose that $M$ admits a metric $\alpha=\sqrt{-1} \alpha_{i \bar{j}} d z^{i} \wedge d \bar{z}^{j}>0$ which is Kähler (that is, $d \alpha=0$ ). Yau's celebrated solution [43] of the Calabi conjecture says that given any smooth positive volume form $\sigma$ on $M$ with $\int_{M} \sigma=\int_{M} \alpha^{n}$, we can find a Kähler metric $\omega$ with this prescribed volume form:

$$
\omega^{n}=\sigma
$$

Moreover, there exists such a metric so that $[\omega]=[\alpha]$ in $H^{2}(M, \mathbb{R})$, and with this cohomological constraint the metric $\omega$ is unique.

Furthermore, Yau's theorem is equivalent to a statement about the first Chern class $c_{1}(M)$. Namely, given any smooth representative $\Psi$ of $c_{1}(M)$, there exists a unique Kähler metric $\omega$ cohomologous to $\alpha$ such that

$$
\operatorname{Ricci}(\omega)=\Psi,
$$

where $\operatorname{Ricci}(\omega)$ is the Ricci form of the Kähler metric $\omega$. Indeed, this follows immediately from the definition of $c_{1}(M)$ and by applying the operator $-\sqrt{-1} \partial \bar{\partial} \log$ to (1.1).

It is natural to investigate whether similar results hold when $M$ does not admit a Kähler metric, but only a Hermitian metric $\alpha$. If we do not impose any constraint on

Supported in part by National Science Foundation grants DMS-1306298 and DMS-1350696 (G. Sz.), DMS-1308988 (V.T.) and DMS-1406164 (B.W.). The second-named author is supported in part by a Sloan Research Fellowship. 
the class of Hermitian metrics that we consider, then (1.1) can be trivially solved by a conformal change of metric. However, there is a natural class of Hermitian metrics which exist on all compact complex manifolds, namely Gauduchon metrics. A Hermitian metric $\alpha$ is called Gauduchon if

$$
\partial \bar{\partial}\left(\alpha^{n-1}\right)=0
$$

and a classical result of Gauduchon [12] says that every Hermitian metric is conformal to a Gauduchon metric (uniquely up to scaling, when $n \geqslant 2$ ). In particular, if we restrict our attention to Gauduchon metrics, then we cannot use non-trivial conformal changes.

Motivated by Yau's theorem, in 1984 Gauduchon [13, §IV.5] posed the following conjecture.

Conjecture 1.1. Let $M$ be a compact complex manifold and $\Psi$ be a closed real $(1,1)$ form on $M$ with $[\Psi]=c_{1}^{\mathrm{BC}}(M) \in H_{\mathrm{BC}}^{1,1}(M, \mathbb{R})$. Then there is a Gauduchon metric $\omega$ on $M$ with

$$
\operatorname{Ricci}(\omega)=\Psi
$$

To explain our notation here,

$$
H_{\mathrm{BC}}^{1,1}(M, \mathbb{R})=\frac{\{d \text {-closed real }(1,1) \text {-forms }\}}{\left\{\sqrt{-1} \partial \bar{\partial} \psi: \psi \in C^{\infty}(M, \mathbb{R})\right\}}
$$

denotes the (finite-dimensional) Bott-Chern cohomology group, and Ricci( $\omega)$ is the ChernRicci form of $\omega$, which is locally given by

$$
\operatorname{Ricci}(\omega)=-\sqrt{-1} \partial \bar{\partial} \log \operatorname{det} g
$$

where we write $\omega=\sqrt{-1} g_{i \bar{j}} d z^{i} \wedge d \bar{z}^{j}$. It is a closed real $(1,1)$-form and its first Bott-Chern cohomology class $c_{1}^{\mathrm{BC}}(M)=[\operatorname{Ricci}(\omega)] \in H_{\mathrm{BC}}^{1,1}(M, \mathbb{R})$ is immediately seen to be independent of the choice of $\omega$.

In the spirit of Yau's theorem, we restate Conjecture 1.1 as an equivalent statement about the existence of Gauduchon metrics with prescribed volume form.

Conjecture 1.2. Let $M$ be a compact complex manifold and $\sigma$ be a smooth positive volume form. Then, there is a Gauduchon metric $\omega$ on $M$ with

$$
\omega^{n}=\sigma
$$

The equivalence with Conjecture 1.1 follows by applying the operator $-\sqrt{-1} \partial \bar{\partial} \log$ to $(1.4)$. 
Our result, Theorem 1.3 below, gives a proof of Conjecture 1.1 (and hence also of Conjecture 1.2). Moreover, our result strengthens the conjecture by imposing a cohomological constraint on the solution $\omega$. Before we state our results, we make some remarks about Conjecture 1.1.

(1) When $M$ is Kähler, this conjecture follows from Yau's theorem.

(2) When $n=2$, the conjecture was proved by Cherrier [4] in 1987 by solving a complex Monge-Ampère equation (see also [38] and [17] for different proofs).

(3) More recently, the second- and third-named authors [40] proved Conjecture 1.1 when $M$ admits an astheno-Kähler metric, i.e. a Hermitian metric $\alpha$ with $\partial \bar{\partial}\left(\alpha^{n-2}\right)=0$ (a condition introduced in [21]).

(4) Clearly, there can be no uniqueness in Conjecture 1.1 as stated.

(5) In [37] the second- and third-named authors proved that given a Hermitian metric $\alpha$, one can always find another Hermitian metric $\omega$ of the form $\omega=\alpha+\sqrt{-1} \partial \bar{\partial} u$, for $u \in C^{\infty}(M, \mathbb{R})$, solving (1.3). If $n=2$, then $\alpha$ Gauduchon implies that $\omega$ is also Gauduchon (and this equation was solved in [4]), but this is no longer the case when $n \geqslant 3$. Hence, the result of [37] does not help to solve Conjecture 1.1 in dimension 3 or higher.

(6) A consequence of Conjecture 1.1 is that $c_{1}^{\mathrm{BC}}(M)=0$ holds if and only if there exist Chern-Ricci-flat Gauduchon metrics on $M$. More information about these "nonKähler Calabi-Yau" manifolds can be found in [35].

We now state our main results. We first introduce some terminology concerning cohomology classes of $(n-1, n-1)$-forms. Define the Aeppli cohomology group

$$
H_{\mathrm{A}}^{n-1, n-1}(M, \mathbb{R})=\frac{\{\partial \bar{\partial} \text {-closed real }(n-1, n-1) \text {-forms }\}}{\left\{\partial \gamma+\bar{\partial} \gamma: \gamma \in \Lambda^{n-2, n-1}(M)\right\}}
$$

This space is naturally in duality with the Bott-Chern cohomology group we considered earlier, with the non-degenerate pairing $H_{\mathrm{A}}^{n-1, n-1}(M, \mathbb{R}) \otimes H_{\mathrm{BC}}^{1,1}(M, \mathbb{R}) \rightarrow \mathbb{R}$ given by wedge product and integration over $M$ (see e.g. [1]). If $\alpha_{0}$ is a Gauduchon metric, then $\alpha_{0}^{n-1}$ defines a class $\left[\alpha_{0}^{n-1}\right] \in H_{\mathrm{A}}^{n-1, n-1}(M, \mathbb{R})$.

We prove the following result.

THEOREM 1.3. Let $M$ be a compact complex manifold with a Gauduchon metric $\alpha_{0}$, and $\Psi$ be a closed real $(1,1)$-form on $M$ with $[\Psi]=c_{1}^{\mathrm{BC}}(M) \in H_{\mathrm{BC}}^{1,1}(M, \mathbb{R})$. Then, there exists a Gauduchon metric $\omega$ satisfying $\left[\omega^{n-1}\right]=\left[\alpha_{0}^{n-1}\right]$ in $H_{\mathrm{A}}^{n-1, n-1}(M, \mathbb{R})$ and

$$
\operatorname{Ricci}(\omega)=\Psi
$$

This result immediately implies Conjectures 1.1 and 1.2. 
In [40], the second- and third-named authors observed that to solve Theorem 1.3 it is enough to solve a certain partial differential equation, which was also independently introduced by Popovici [30]. This equation is a variant of one introduced by $\mathrm{Fu}-\mathrm{Wang}-$ $\mathrm{Wu}[10]$ and related to Harvey-Lawson's notion of $(n-1)$-plurisubharmonic functions [18], [19].

Namely, we seek a Hermitian metric $\omega$ on $M$ with the property that

$$
\omega^{n-1}=\alpha_{0}^{n-1}+\partial \gamma+\overline{\partial \gamma},
$$

where

$$
\gamma=\frac{1}{2} \sqrt{-1} \bar{\partial} u \wedge \alpha^{n-2},
$$

$u \in C^{\infty}(M, \mathbb{R})$ and $\alpha$ is a background Gauduchon metric. Clearly, by construction, the metric $\omega$ is Gauduchon assuming that $\alpha_{0}$ is Gauduchon. Substituting, we see that

$$
\omega^{n-1}=\alpha_{0}^{n-1}+\sqrt{-1} \partial \bar{\partial} u \wedge \alpha^{n-2}+\operatorname{Re}\left(\sqrt{-1} \partial u \wedge \bar{\partial}\left(\alpha^{n-2}\right)\right),
$$

while if we write

$$
\operatorname{Ricci}(\omega)=\Psi+\sqrt{-1} \partial \bar{\partial} F
$$

then (1.5) is equivalent to

$$
\omega^{n}=e^{F+b} \alpha^{n},
$$

for some constant $b \in \mathbb{R}$. This is exactly the equation that we solve, thus resolving [ 40 , Conjecture 1.5] and [30, Question 1.2].

THEOREM 1.4. Let $M$ be a compact complex manifold with $\operatorname{dim}_{\mathbb{C}} M=n \geqslant 2$, equipped with a Hermitian metric $\alpha_{0}$ and a Gauduchon metric $\alpha$. Given a smooth function $F$ on $M$, we can find a unique $u \in C^{\infty}(M, \mathbb{R})$ with $\sup _{M} u=0$, and a unique $b \in \mathbb{R}$ such that the Hermitian metric $\omega$ defined by

$$
\omega^{n-1}:=\alpha_{0}^{n-1}+\sqrt{-1} \partial \bar{\partial} u \wedge \alpha^{n-2}+\operatorname{Re}\left(\sqrt{-1} \partial u \wedge \bar{\partial}\left(\alpha^{n-2}\right)\right)>0
$$

satisfies

$$
\omega^{n}=e^{F+b} \alpha^{n} .
$$

Clearly, as we just described, Theorem 1.3 follows from this result if we take $\alpha_{0}=\alpha$ Gauduchon. We make some remarks about Theorem 1.4.

(1) In the case when $\alpha$ is Kähler, or more generally if the linear term involving $\partial u$ is removed, the equation (1.7) reduces to the Monge-Ampère equation for $(n-1)$ plurisubharmonic functions, solved by the second- and third-named authors [39], [40] (see also [11] for earlier partial results). 
(2) In the case when $n=2$, this equation reduces to the complex Monge-Ampère equation solved in [4] (see also [38]).

(3) It was shown in [40] that Theorem 1.4 can be reduced to a second-order a-priori estimate of the form (cf. [20])

$$
\sup _{M}|\sqrt{-1} \partial \bar{\partial} u|_{\alpha} \leqslant C\left(1+\sup _{M}|\nabla u|_{\alpha}^{2}\right)
$$

for solutions $u$ of (1.7). This is precisely the estimate we prove in this paper.

(4) If in Theorem 1.4 we assume that $\alpha_{0}$ is strongly Gauduchon in the sense of Popovici [29], namely that $\bar{\partial}\left(\alpha_{0}^{n-1}\right)$ is $\partial$-exact, then, by construction, so is the solution $\omega$. Thus, we also get a Calabi-Yau-type theorem for strongly Gauduchon metrics. More applications of this theorem can be found in [30].

(5) Our method of proof of Theorem 1.4 can also be used to solve an equation introduced by $\mathrm{Fu}$-Wang-Wu [10] in certain cases. Suppose we have a compact Hermitian manifold $\left(M, \alpha_{0}\right)$ and we seek a Hermitian metric $\omega$ solving (1.7) with the property that

$$
\omega^{n-1}=\alpha_{0}^{n-1}+\sqrt{-1} \partial \bar{\partial}\left(u \alpha^{n-2}\right)
$$

for some $u \in C^{\infty}(M, \mathbb{R})$ and some Hermitian metric $\alpha$. This setup is particularly interesting because if $\alpha_{0}$ is balanced (i.e. $d\left(\alpha_{0}^{n-1}\right)=0$, see [26]), then so is $\omega$, and one obtains a Calabi-Yau theorem for balanced metrics (see also [35, §4]). When $\alpha$ is Kähler, this setup reduces to the setting of item (1). If we instead assume that $\alpha$ is astheno-Kähler, then we see that

$$
\omega^{n-1}=\alpha_{0}^{n-1}+\sqrt{-1} \partial \bar{\partial} u \wedge \alpha^{n-2}+2 \operatorname{Re}\left(\sqrt{-1} \partial u \wedge \bar{\partial}\left(\alpha^{n-2}\right)\right),
$$

which differs from (1.6) just for a factor of 2. Therefore, this problem falls into our general framework (see Theorem 2.2 below), and we conclude that we have uniform a-priori estimates for solutions of this equation. The exact same argument as in [40, Theorem 1.7] using the continuity method then shows that the equation is indeed solvable. In the case when we choose $\alpha_{0}$ to be balanced, this gives a proof of [35, Conjectures 4.1 and 4.2], assuming that $M$ admits astheno-Kähler metrics. However, we should remark that we are not aware of any example of a non-Kähler compact complex manifold which admits both balanced and astheno-Kähler metrics. $\left.{ }^{1}\right)$

(6) The same argument as the proof of Theorem 1.4 also allows us to find a Gauduchon metric $\omega$ solving the "complex-Hessian" equation

$$
\omega^{k} \wedge \alpha^{n-k}=e^{F+b} \alpha^{n}
$$

$\left({ }^{1}\right)$ After this paper was posted, and prompted by our remark, explicit examples were constructed in [9] and [22] in all complex dimensions $\geqslant 4$. 
for any $1 \leqslant k \leqslant n$; see also [34, Proposition 24] for the case of $(n-1)$-plurisubharmonic functions, and [8], [20] for the standard Kähler case where $\omega=\alpha+\sqrt{-1} \partial \bar{\partial} u$.

(7) The complex setting is very different from the real analogue of (1.7), treated for example in more generality in [16]. The underlying reason is the fact that there are two different types of complex derivatives. In our case, the special structure of the gradient term in (1.7) plays a key role.

In fact, Theorem 1.4 follows from a much more general result, where we consider a large class of fully non-linear second-order elliptic equations on Hermitian manifolds. This result is analogous to the main result in [34], giving a-priori estimates in the presence of a suitable subsolution. We will state this as Theorem 2.2 in $\S 2$. This result fits into a large body of work on fully non-linear second-order elliptic equations, going back to the work of Caffarelli-Nirenberg-Spruck [3] on the Dirichlet problem on domains in $\mathbb{R}^{n}$. Some other works on this topic include [6], [7], [14]-[17], [23]-[25], [27], [28], [32], [33], [41], [42], [44]-[46].

In our proof of Theorem 2.2, we use some of the language and approaches of the recent paper of the first-named author [34]. However, if one is only interested in a direct proof of Theorem 1.4, one can equally well use the language of [40]. In any case, the key new ingredient is an understanding of the structure of the term $\operatorname{Re}\left(\sqrt{-1} \partial u \wedge \bar{\partial}\left(\alpha^{n-2}\right)\right)$.

The paper is organized as follows. In $\S 2$ we will introduce some notation and state our main technical result (Theorem 2.2). The proof of this theorem will be given in $\S 3$, and in $\S 4$ we show how this implies Theorem 1.4.

As the present work neared completion, we were informed that Bo Guan and Xiaolan Nie have a work in progress on related results.

Acknowledgments. We thank the referee for useful remarks.

\section{Background and the general setting}

Let $(M, \alpha)$ be a compact Hermitian manifold of complex dimension $n$ and write

$$
\alpha=\sqrt{-1} \alpha_{i \bar{j}} d z^{i} \wedge d \bar{z}^{j}>0 .
$$

Fix a background $(1,1)$-form $\chi=\sqrt{-1} \chi_{i \bar{j}} d z^{i} \wedge d \bar{z}^{j}$ which is not necessarily positive definite. Let $W_{i \bar{j}}(\nabla u)$ be a Hermitian tensor which depends linearly on $\nabla u$. For $u: M \rightarrow \mathbb{R}$, define a new tensor $g_{i \bar{j}}$ by

$$
g_{i \bar{j}}:=\chi_{i \bar{j}}+u_{i \bar{j}}+W_{i \bar{j}} .
$$

Note that we do not assume that $\left(g_{i \bar{j}}\right)$ is positive definite. We will study equations for $g$, where $W$ has a special structure related to the equation (1.7). To define this, let us 
write

$$
\tilde{g}_{i \bar{j}}:=P_{\alpha}\left(g_{i \bar{j}}\right)=\frac{1}{n-1}\left(\left(\operatorname{tr}_{\alpha} g\right) \alpha_{i \bar{j}}-g_{i \bar{j}}\right),
$$

where $P_{\alpha}$ is an operator on tensors, depending on the fixed metric $\alpha$, defined by the second equality in (2.2). Here $\operatorname{tr}_{\alpha} g$ is the trace of $g$ with respect to $\alpha$. As an aside, if $\alpha$ is the Euclidean metric on $\mathbb{C}^{n}$, then the condition $P_{\alpha}\left(u_{i \bar{j}}\right) \geqslant 0$ is equivalent to saying that $u$ is $(n-1)$-plurisubharmonic, in the sense of Harvey-Lawson [18].

Observe that, writing $\Delta=\alpha^{k \bar{\ell}} \partial_{k} \partial_{\bar{\ell}}$,

$$
\tilde{g}_{i \bar{j}}=\tilde{\chi}_{i \bar{j}}+\frac{1}{n-1}\left((\Delta u) \alpha_{i \bar{j}}-u_{i \bar{j}}\right)+Z_{i \bar{j}}
$$

for $Z$ given by

$$
Z_{i \bar{j}}:=P_{\alpha}\left(W_{i \bar{j}}\right)=\frac{1}{n-1}\left(\left(\operatorname{tr}_{\alpha} W\right) \alpha_{i \bar{j}}-W_{i \bar{j}}\right),
$$

and similarly, $\tilde{\chi}_{i \bar{j}}=P_{\alpha}\left(\chi_{i \bar{j}}\right)$. Note that we can also write $W$ explicitly in terms of $Z$ :

$$
W_{i \bar{j}}=\left(\operatorname{tr}_{\alpha} Z\right) \alpha_{i \bar{j}}-(n-1) Z_{i \bar{j}}
$$

A crucial assumption we make is that $W$ depends on $\nabla u$ in the following way: we assume that the tensor $Z$ has the form

$$
Z_{i \bar{j}}=Z_{i \bar{j}}^{p} u_{p}+\bar{Z}_{j \bar{i}}^{p} u_{p}
$$

for some tensor $Z_{i \bar{j}}^{p}$ independent of $u$. In addition we have the following.

Assumption for $W$. In orthonormal coordinates for $\alpha$ at any given point, the component $Z_{i \bar{j}}$ is independent of $u_{\bar{i}}$ and $u_{j}$ (in other words, $Z_{i \bar{j}}^{j}=0$ for all $i$ and $j$ ), and $\nabla_{i} Z_{i \bar{i}}$ is independent of $u_{\bar{i}}$ (in other words, $\nabla_{\bar{i}} Z_{i \bar{i}}^{i}=0$ for all $i$ ).

Here, $\nabla$ is the Chern connection of $\alpha$. This assumption expresses a certain skewsymmetry requirement for the tensor $W$. This assumption is satisfied for the $(n-1)$ plurisubharmonic Monge-Ampère equation, the case of most interest to us, see (4.3) below, the key reason being that the torsion tensor is skew-symmetric.

Let us record here a few consequences of this assumption, which will be used later. Taking $\nabla_{p}$ of $(2.6)$, setting $i=j$, evaluating at that point and using that $Z_{i \bar{i}}^{i}=0$, we see that $\nabla_{p} Z_{i \bar{i}}$ is independent of $u_{i i}$ and $u_{i \bar{i}}$ (at that point, in orthonormal coordinates for $\alpha$ ). Here, the subscripts of $u$ denote ordinary partial derivatives. Similarly, $\nabla_{i} Z_{p \bar{i}}$ is independent of $u_{i i}$. Taking two covariant derivatives, we have

$$
\begin{aligned}
\nabla_{\bar{i}} \nabla_{i} Z_{i \bar{i}}=\nabla_{\bar{i}} \nabla_{i} Z_{i \bar{i}}^{p} u_{p}+Z_{i \bar{i}}^{p} \nabla_{\bar{i}} \nabla_{i} u_{p}+\nabla_{i} Z_{i \bar{i}}^{p} \nabla_{\bar{i}} u_{p}+\nabla_{\bar{i}} Z_{i \bar{i}}^{p} \nabla_{i} u_{p} \\
+\nabla_{\bar{i}} \nabla_{i} \overline{Z_{i \bar{i}}^{p}} u_{\bar{p}}+\overline{Z_{i \bar{i}}^{p}} \nabla_{\bar{i}} \nabla_{i} u_{\bar{p}}+\nabla_{i} \overline{Z_{i \bar{i}}^{p}} \nabla_{\bar{i}} u_{\bar{p}}+\nabla_{\bar{i}} \overline{Z_{i \bar{i}}^{p}} \nabla_{i} u_{\bar{p}}
\end{aligned}
$$


and evaluating at our point and using the assumptions $\nabla_{\bar{i}} Z_{\bar{i}}^{i}=0$ and $Z_{i \bar{i}}^{i}=0$, we see that $\nabla_{\bar{i}} \nabla_{i} Z_{i \bar{i}}$ is independent of $u_{i i}, u_{\bar{i} \bar{i}}, u_{i i \bar{i}}$ and $u_{i \bar{i} \bar{i}}$.

Given a smooth function $h$, we study equations of the form

$$
F(A)=h,
$$

where $A$ is the endomorphism $A_{j}^{i}=\alpha^{i \bar{p}} g_{j \bar{p}}$ of the holomorphic tangent bundle, which is Hermitian with respect to the inner product defined by $\alpha$, and $F(A)$ is a symmetric function of the eigenvalues $\lambda_{1}, \ldots, \lambda_{n}$ of $A$ :

$$
F(A)=f\left(\lambda_{1}, \ldots, \lambda_{n}\right) .
$$

We assume that our operator $F$ has the special form $F(M)=\widetilde{F}(P(M))$, where

$$
P(M)=(n-1)^{-1}(\operatorname{Tr}(M) I-M),
$$

analogous to $P_{\alpha}$ above (here writing $\operatorname{Tr}(M)$ for the trace of the matrix $M$ ), and

$$
\widetilde{F}(B)=\tilde{f}\left(\mu_{1}, \ldots, \mu_{n}\right),
$$

where $\mu_{1}, \ldots, \mu_{n}$ are the eigenvalues of $B$, and $\tilde{f}$ is another symmetric function. In terms of eigenvalues, this means that

$$
f\left(\lambda_{1}, \ldots, \lambda_{n}\right)=(\tilde{f} \circ P)\left(\lambda_{1}, \ldots, \lambda_{n}\right),
$$

where we are writing $P$ for the map $\mathbb{R}^{n} \rightarrow \mathbb{R}^{n}$ induced on diagonal matrices by the matrix map $P$ above. Explicitly, writing $\mu=P(\lambda)$ for the corresponding $n$-tuples $\lambda, \mu \in \mathbb{R}^{n}$, we have

$$
f\left(\lambda_{1}, \ldots, \lambda_{n}\right)=\tilde{f}\left(\mu_{1}, \ldots, \mu_{n}\right), \quad \text { for } \mu_{k}=\frac{1}{n-1} \sum_{i \neq k} \lambda_{i} .
$$

Assumptions for $\tilde{f}$ and $h$. We make the following assumptions on $\tilde{f}$, and the function $h$ in our equation:

(i) $\tilde{f}$ is defined on an open symmetric convex cone $\widetilde{\Gamma} \varsubsetneqq \mathbb{R}^{n}$, containing the positive orthant $\Gamma_{n}=\left\{\left(x_{1}, \ldots, x_{n}\right) \in \mathbb{R}^{n}: x_{i}>0, i=1, \ldots, n\right\}$.

(ii) $\tilde{f}$ is symmetric, smooth, concave and increasing, i.e. its partials satisfy $\tilde{f}_{i}>0$ for all $i$.

(iii) $\sup _{\partial \widetilde{\Gamma}} \tilde{f}<\inf _{M} h$.

(iv) For all $\mu \in \widetilde{\Gamma}$, we have

$$
\lim _{t \rightarrow \infty} \tilde{f}(t \mu)=\sup _{\widetilde{\Gamma}} \tilde{f},
$$

where both sides are allowed to be $\infty$.

(v) $h$ is a smooth function on $M$. 
Define the cone $\Gamma \subset \mathbb{R}^{n}$ by $\Gamma=P^{-1}(\widetilde{\Gamma})$. Observe that $P$ maps $\Gamma_{n}$ into $\Gamma_{n}$. It is then easy to see that the function $f=\tilde{f} \circ P: \Gamma \rightarrow \mathbb{R}$ satisfies exactly the same conditions as $\tilde{f}$. In particular, some of the results of [34] can be applied to the equation $F(A)=h$. We need the following definition (see [34, Remark 8] to see the equivalence with the definition there), which is a modification of a notion introduced by Guan [14].

Definition 2.1. We say that $u$ is a $\mathcal{C}$-subsolution for the equation $F(A)=h$ if the following holds. Let $g_{i \bar{j}}$ be defined as in (2.1). We require that for every point $x \in M$, if $\lambda=\left(\lambda_{1}, \ldots, \lambda_{n}\right)$ denote the eigenvalues of the endomorphism $\alpha^{i \bar{p}} g_{j \bar{p}}$ at $x$, then for all $i=1, \ldots, n$ we have

$$
\lim _{t \rightarrow \infty} f\left(\lambda+t \mathbf{e}_{i}\right)>h(x)
$$

Here $\mathbf{e}_{i}$ denotes the $i$ th standard basis vector. Note that part of the requirement is that $\lambda+t \mathbf{e}_{i} \in \Gamma$ for sufficiently large $t$, for the limit to be defined.

With this background, our main estimate is the following, analogous to the main result in [34]. We will give the proof in $\S 3$.

THEOREM 2.2. Suppose that $\underline{u}$ is a $\mathcal{C}$-subsolution for the equation $F(A)=h$, and $u$ is a smooth solution, normalized by $\sup _{M} u=0$. Suppose that $F$ and $h$ satisfy the assumptions above, including the assumption for the gradient term $W$. Then, for each $k=0,1,2, \ldots$, we have an estimate $\|u\|_{C^{k}(M, \alpha)} \leqslant C_{k}$, with constant $C_{k}$ depending on $k$, on the background data $M, \alpha, \chi, F, h$, the coefficients of $W$ and the subsolution $\underline{u}$.

The case of primary interest for us is equation (1.7), which corresponds to the symmetric function

$$
\tilde{f}\left(\mu_{1}, \ldots, \mu_{n}\right)=\log \left(\mu_{1} \ldots \mu_{n}\right)
$$

on the positive orthant $\widetilde{\Gamma}=\Gamma_{n}$. It is straightforward to check that $\tilde{f}$ satisfies the conditions above. Indeed, $\tilde{f}$ converges to $-\infty$ on the boundary $\partial \Gamma_{n}$, so (iii) is satisfied, and for (iv) it is enough to note that $\tilde{f}(t \mu)=\tilde{f}(\mu)+n \log t$, which converges to $\infty$ as $t \rightarrow \infty$.

In addition, if $\mu \in \Gamma_{n}$, then we also have

$$
\lim _{t \rightarrow \infty} \tilde{f}\left(\mu+t \mathbf{e}_{i}\right)=\infty
$$

for all $i$. This means that for a function $u$ to be a $\mathcal{C}$-subsolution for this equation, the only requirement is that, at each point, the eigenvalues $\lambda$ of $\alpha^{i \bar{p}} g_{j \bar{p}}$ satisfy $P(\lambda) \in \Gamma_{n}$. In other words, the requirement is that $\tilde{g}_{i \bar{j}}$, defined in (2.2), is positive definite.

Note that, if $\underline{u}$ is a $\mathcal{C}$-subsolution, then replacing $\chi$ by

$$
\chi_{i \bar{j}}^{\prime}=\chi_{i \bar{j}}+\underline{u}_{i \bar{j}}+W_{i \bar{j}}(\nabla \underline{u}),
$$

we may assume that $\underline{u}=0$. The important consequence of 0 being a $\mathcal{C}$-subsolution is the following, which follows from [34, Proposition 6 and Lemma 9]. 
Proposition 2.3. Suppose that 0 is a $\mathcal{C}$-subsolution for the equation $F(A)=h$, and $u$ is a solution. Define $g_{i \bar{j}}$ as in (2.1). There are constants $R, \varkappa>0$, independent of $u$, with the following property. Let $x \in M$, and choose orthonormal coordinates for $\alpha$ at $x$, such that $g$ is diagonal, with eigenvalues $\lambda=\left(\lambda_{1}, \ldots, \lambda_{n}\right)$. If $|\lambda|>R$, then there are two possibilities:

(a) we either have

$$
\sum_{k} f_{k}(\lambda)\left[\chi_{k \bar{k}}-\lambda_{k}\right]>\varkappa \sum_{k} f_{k}(\lambda)
$$

(b) or $f_{k}(\lambda)>\varkappa \sum_{i} f_{i}(\lambda)$ for all $k$.

In addition $\sum_{k} f_{k}(\lambda)>\varkappa$.

We collect some other basic properties of the functions $f$ and $\tilde{f}$. Suppose that $\lambda \in \Gamma$ with $\lambda_{1} \geqslant \ldots \geqslant \lambda_{n}$. Then $\mu_{1} \leqslant \ldots \leqslant \mu_{n}$, and so, by property (ii), $\tilde{f}_{1} \geqslant \ldots \geqslant \tilde{f}_{n}>0$ (see e.g. [34, p. 12]). We have

$$
f_{k}=\frac{1}{n-1} \sum_{i \neq k} \tilde{f}_{i}
$$

which implies that $0<f_{1} \leqslant \ldots \leqslant f_{n}$. Also, for $k>1$,

$$
0<\frac{\tilde{f}_{1}}{n-1} \leqslant f_{k} \leqslant \tilde{f}_{1},
$$

i.e. the $f_{k}$ for $k>1$ are all comparable, while $f_{1}$ may be relatively small. In addition, from (2.11) with $k=1$, we obtain

$$
\tilde{f}_{i} \leqslant(n-1) f_{1}, \quad \text { for } i>1 \text {. }
$$

Proposition 2.3 is easy to verify directly in the case of equation (1.7), where

$$
f(\lambda)=\log \left(\mu_{1} \ldots \mu_{n}\right)
$$

with $\mu_{k}$ defined as in (2.9). Indeed, in this case

$$
\tilde{f}_{i}(\mu)=\frac{1}{\mu_{i}}
$$

and

$$
f_{k}(\lambda)=\frac{1}{n-1} \sum_{i \neq k} \frac{1}{\mu_{i}}
$$

The function 0 being a $\mathcal{C}$-subsolution means that $\tilde{\chi}$ in $(2.3)$ is positive definite. We have

$$
\sum_{k} f_{k}(\lambda) \chi_{k \bar{k}}=\sum_{i} \frac{1}{\mu_{i}} \widetilde{\chi}_{i \bar{i}}>\tau \sum_{i} \frac{1}{\mu_{i}}=\tau \sum_{k} f_{k}(\lambda),
$$


for some $\tau>0$ depending on a lower bound for $\tilde{\chi}$. We also have

$$
\sum_{k} \lambda_{k} f_{k}(\lambda)=n
$$

It follows that we have the alternative (a) in Proposition 2.3 whenever there is one sufficiently small $\mu_{i}$, which by the equation $f(\lambda)=h$ is equivalent to having at least one large $\mu_{i}$, i.e. at least one large $\lambda_{i}$. In addition,

$$
\sum_{k} f_{k}(\lambda)=\sum_{i=1}^{n} \frac{1}{\mu_{i}} \geqslant n\left(\mu_{1} \ldots \mu_{n}\right)^{-1 / n}=n e^{-h / n},
$$

so that the final claim in Proposition 2.3 also holds.

\section{Proof of the main estimate}

In this section, we give the proof of Theorem 2.2.

First of all, a uniform bound $\|u\|_{L^{\infty}(M)} \leqslant C$ can be obtained by a simple modification of the argument in [34, Proposition 10 and Remark 12], which is itself inspired by Błocki's proof of the $L^{\infty}$ estimate in Yau's theorem [2]. In the setting of equation (1.7), the $L^{\infty}$ estimate of $u$ was first proved in [40], using a different method more analogous to the arguments in [4], [37], [39], [43].

Our main goal is the following estimate:

$$
\sup _{M}|\sqrt{-1} \partial \bar{\partial} u|_{\alpha} \leqslant C\left(\sup _{M}|\nabla u|_{\alpha}^{2}+1\right),
$$

for a constant $C$ depending only on the fixed data of Theorem 2.2. We remark that an estimate of this form was proved in the context of the complex Hessian equations by Hou$\mathrm{Ma}-\mathrm{Wu}$ [20], making use of ideas of Chou-Wang [5]. For the (n-1)-plurisubharmonic equation (namely, equation (1.7) without the linear term in $\partial u$ ), an estimate of this type was proved in [39], [40]. This was then generalized much further in [34], where the estimate was shown to hold for a large class of equations. Our proof begins along similar lines to these papers. The new difficulty comes from the linear term in $\partial u$, which, fortunately, has a special structure that we can exploit.

In fact, the estimate (3.1) is equivalent to the bound

$$
\lambda_{1} \leqslant C K,
$$

where $K=1+\sup _{M}|\nabla u|_{\alpha}^{2}$ and $\lambda_{1}$ is the largest eigenvalue of $A=\left(A_{j}^{i}\right)=\left(\alpha^{i \bar{p}} g_{j \bar{p}}\right)$. Indeed, our assumption on the cone $\Gamma$ implies that $\sum_{i} \lambda_{i}>0$ (see Caffarelli-Nirenberg-Spruck [3]). 
Then, if $\lambda_{1}$ is bounded from above by $C K$, so is $\left|\lambda_{i}\right|$ for all $i$, giving the same bound for $\sup _{M}|\sqrt{-1} \partial \bar{\partial} u|_{\alpha}$.

We consider the function

$$
H=\log \lambda_{1}+\phi\left(|\nabla u|_{\alpha}^{2}\right)+\psi(u)
$$

where $\phi$ is defined by

$$
\phi(t)=-\frac{1}{2} \log \left(1-\frac{t}{2 K}\right),
$$

so that $\phi\left(|\nabla u|_{\alpha}^{2}\right) \in\left[0, \frac{1}{2} \log 2\right]$ satisfies

$$
\frac{1}{4 K}<\phi^{\prime}<\frac{1}{2 K}, \quad \phi^{\prime \prime}=2\left(\phi^{\prime}\right)^{2}>0
$$

and $\psi$ is defined by

$$
\psi(t)=D_{1} e^{-D_{2} t},
$$

for sufficiently large uniform constants $D_{1}, D_{2}>0$ to be chosen later. By the $L^{\infty}$ bound on $u$, the quantity $\psi(u)$ is uniformly bounded.

We remark that we follow [34] by computing with the largest eigenvalue $\lambda_{1}$ instead of the analogous quantity in [40], but in fact either quantity works, at least in the case of equation (1.7). Also note that, while the function $\phi$ here coincides with that in [20] (and also in [39], [40]), our choice of $\psi$ is crucially different.

We work at a point where $H$ achieves its maximum, in orthonormal complex coordinates for $\alpha$ centered at this point, such that $g$ is diagonal and $\lambda_{1}=g_{1 \overline{1}}$. The quantity $H$ need not be smooth at this maximum point, because the largest eigenvalue of $A$ may have eigenspace of dimension larger than 1 . To take care of this, we carry out a perturbation argument as in [34], choosing local coordinates such that $H$ achieves its maximum at the origin, where $A$ is diagonal with eigenvalues $\lambda_{1} \geqslant \ldots \geqslant \lambda_{n}$, as before. We fix a diagonal matrix $B$, with $B_{1}^{1}=0$ and $0<B_{2}^{2}<\ldots<B_{n}^{n}$, and we define $\tilde{A}=A-B$, denoting its eigenvalues by $\tilde{\lambda}_{1}, \ldots, \tilde{\lambda}_{n}$.

At the origin, we have

$$
\tilde{\lambda}_{1}=\lambda_{1} \quad \text { and } \quad \tilde{\lambda}_{i}=\lambda_{i}-B_{i}^{i}, \quad i>1
$$

and $\tilde{\lambda}_{1}>\tilde{\lambda}_{2}>\ldots>\tilde{\lambda}_{n}$. As discussed above, our assumption on the cone $\Gamma$ implies that $\sum_{i} \lambda_{i}>0$, and we fix the matrix $B$ small enough so that

$$
\sum_{i} \tilde{\lambda}_{i}>-1
$$


We can choose this matrix $B$ in such a way that, in addition,

$$
\sum_{p>1} \frac{1}{\lambda_{1}-\tilde{\lambda}_{p}} \leqslant C,
$$

for some fixed constant $C$ depending on the dimension $n$. Now, after possibly shrinking the chart, the quantity

$$
\widetilde{H}=\log \tilde{\lambda}_{1}+\phi\left(|\nabla u|_{\alpha}^{2}\right)+\psi(u)
$$

is smooth on the chart and achieves its maximum at the origin. We will apply the maximum principle to $\widetilde{H}$. Our goal is to obtain the bound $\tilde{\lambda}_{1} \leqslant C K$ at the origin, which will give us the required estimate (3.1). Hence, we may and do assume that $\tilde{\lambda}_{1} \gg K$ at this point.

We now differentiate $\widetilde{H}$ at the origin and, as before, we use subscripts $k$ and $\bar{\ell}$ to denote the partial derivatives $\partial / \partial z^{k}$ and $\partial / \partial \bar{z}^{\ell}$. We have

$$
\begin{aligned}
\widetilde{H}_{k} & =\frac{\tilde{\lambda}_{1, k}}{\tilde{\lambda}_{1}}+\phi^{\prime}\left(\alpha^{p \bar{q}} u_{p} u_{\bar{q} k}+\alpha^{p \bar{q}} u_{p k} u_{\bar{q}}+\left(\alpha^{p \bar{q}}\right)_{k} u_{p} u_{\bar{q}}\right)+\psi^{\prime} u_{k} \\
& =\frac{\tilde{\lambda}_{1, k}}{\tilde{\lambda}_{1}}+\phi^{\prime}\left(u_{p} u_{\bar{p} k}+u_{p k} u_{\bar{p}}+\left(\alpha^{p \bar{q}}\right)_{k} u_{p} u_{\bar{q}}\right)+\psi^{\prime} u_{k}=\frac{\tilde{\lambda}_{1, k}}{\tilde{\lambda}_{1}}+\phi^{\prime} V_{k}+\psi^{\prime} u_{k},
\end{aligned}
$$

where $V_{k}:=u_{p} u_{\bar{p} k}+u_{p k} u_{\bar{p}}+\left(\alpha^{p \bar{q}}\right)_{k} u_{p} u_{\bar{q}}$. Differentiating once more,

$$
\begin{aligned}
\widetilde{H}_{k \bar{k}}= & \frac{\tilde{\lambda}_{1, k \bar{k}}}{\lambda_{1}}-\frac{\left|\tilde{\lambda}_{1, k}\right|^{2}}{\lambda_{1}^{2}}+\phi^{\prime}\left(u_{p} u_{\bar{p} k \bar{k}}+u_{\bar{p}} u_{p k \bar{k}}+\sum_{p}\left|u_{p k}\right|^{2}+\sum_{p}\left|u_{\bar{p} k}\right|^{2}\right. \\
& \left.+\left(\alpha^{p \bar{q}}\right)_{\bar{k}} u_{p} u_{\bar{q} k}+\left(\alpha^{p \bar{q}}\right)_{\bar{k}} u_{p k} u_{\bar{q}}+\left(\alpha^{p \bar{q}}\right)_{k \bar{k}} u_{p} u_{\bar{q}}+\left(\alpha^{p \bar{q}}\right)_{k}\left(u_{p} u_{\bar{q} \bar{k}}+u_{p \bar{k}} u_{\bar{q}}\right)\right) \\
& +\phi^{\prime \prime}\left|V_{k}\right|^{2}+\psi^{\prime \prime}\left|u_{k}\right|^{2}+\psi^{\prime} u_{k \bar{k}},
\end{aligned}
$$

where we use the convention that we sum in all repeated indices except the free index $k$.

Since $1 / 4 K<\phi^{\prime}<1 / 2 K$, we can absorb all the terms involving $\alpha$ into the squared terms up to a constant, i.e. we have

$$
\begin{aligned}
\tilde{H}_{k \bar{k}} \geqslant & \frac{\tilde{\lambda}_{1, k \bar{k}}}{\lambda_{1}}-\frac{\left|\tilde{\lambda}_{1, k}\right|^{2}}{\lambda_{1}^{2}}+\phi^{\prime}\left(u_{p} u_{\bar{p} k \bar{k}}+u_{\bar{p}} u_{p k \bar{k}}\right)+\frac{1}{5 K} \sum_{p}\left(\left|u_{p k}\right|^{2}+\left|u_{\bar{p} k}\right|^{2}\right) \\
& +\phi^{\prime \prime}\left|V_{k}\right|^{2}+\psi^{\prime \prime}\left|u_{k}\right|^{2}+\psi^{\prime} u_{k \bar{k}}-C .
\end{aligned}
$$

The constant $C$ denotes a constant that may change from line to line, but it does not depend on the parameters $D_{1}$ and $D_{2}$ that we are yet to choose. 


\section{- Calculation of $\tilde{\lambda}_{1, k \bar{k}}$}

Let us now compute the derivatives of $\tilde{\lambda}_{1}$. We have the following general formulas for the derivatives of the eigenvalue $\lambda_{i}$ of complex $n \times n$ matrices at a diagonal matrix with

distinct real eigenvalues (see for instance Spruck [31] in the case of matrices with real entries):

$$
\begin{aligned}
\lambda_{i}^{p q} & =\delta_{p i} \delta_{q i} \\
\lambda_{i}^{p q, r s} & =\left(1-\delta_{i p}\right) \frac{\delta_{i q} \delta_{i r} \delta_{p s}}{\lambda_{i}-\lambda_{p}}+\left(1-\delta_{i r}\right) \frac{\delta_{i s} \delta_{i p} \delta_{r q}}{\lambda_{i}-\lambda_{r}},
\end{aligned}
$$

where $\lambda_{i}^{p q}$ denotes the derivative with respect to the $(p, q)$-entry $A_{q}^{p}$ of the matrix $A$, as a complex variable.

Denoting by $\tilde{\lambda}_{1}$ the largest eigenvalue of the endomorphism $\tilde{A}$ again, we have, using (3.6),

$$
\tilde{\lambda}_{1, k}=\tilde{\lambda}_{1}^{p q} \nabla_{k}\left(\tilde{A}_{q}^{p}\right)=\nabla_{k}\left(\tilde{A}_{1}^{1}\right)=\nabla_{k} g_{1 \overline{1}}-\nabla_{k} B_{1}^{1}=g_{1 \overline{1} k}+\left(\alpha^{1 \overline{1}}\right)_{k} g_{1 \overline{1}}
$$

since $\nabla_{k} B_{1}^{1}=0$ at the origin. Here we computed using covariant derivatives with respect to the Chern connection of $\alpha$, which makes the positivity of certain terms more apparent when we take second derivatives:

$$
\tilde{\lambda}_{1, k \bar{k}}=\tilde{\lambda}_{1}^{p q} \nabla_{\bar{k}} \nabla_{k} \tilde{A}_{q}^{p}+\tilde{\lambda}_{1}^{p q, r s}\left(\nabla_{k} \tilde{A}_{q}^{p}\right)\left(\nabla_{\bar{k}} \tilde{A}_{s}^{r}\right)=\nabla_{\bar{k}} \nabla_{k} g_{1 \overline{1}}+\tilde{\lambda}_{1}^{p q, r s}\left(\nabla_{k} \tilde{A}_{q}^{p}\right)\left(\nabla_{\bar{k}} \tilde{A}_{s}^{r}\right),
$$

where we used (3.6) and the fact that $\nabla_{\bar{k}} \nabla_{k} B_{1}^{1}=0$ at the origin. To rewrite this in terms of partial derivatives, note first that

$$
\begin{aligned}
\nabla_{k} g_{1 \overline{1}} & =g_{1 \overline{1} k}-\Gamma_{k 1}^{m} g_{m \overline{1}} \\
\nabla_{\bar{k}} \nabla_{k} g_{1 \overline{1}} & =g_{1 \overline{1} k \bar{k}}-\left(\partial_{\bar{k}} \Gamma_{k 1}^{m}\right) g_{m \overline{1}}-\Gamma_{k 1}^{m} g_{m \overline{1} \bar{k}}-\overline{\Gamma_{k 1}^{q}} g_{1 \bar{q} k}+\overline{\Gamma_{k 1}^{q}} \Gamma_{k 1}^{m} g_{m \bar{q}} \\
& =g_{1 \overline{1} k \bar{k}}+O\left(\sum_{m}\left|g_{1 \bar{m} k}\right|+\lambda_{1}\right) .
\end{aligned}
$$

In addition, we have

$$
\begin{aligned}
\tilde{\lambda}_{1}^{p q, r s}\left(\nabla_{k} \tilde{A}_{q}^{p}\right)\left(\nabla_{\bar{k}} \tilde{A}_{s}^{r}\right) & =\sum_{p>1} \frac{\left(\nabla_{k} \tilde{A}_{1}^{p}\right)\left(\nabla_{\bar{k}} \tilde{A}_{p}^{1}\right)+\left(\nabla_{k} \tilde{A}_{p}^{1}\right)\left(\nabla_{\bar{k}} \tilde{A}_{1}^{p}\right)}{\lambda_{1}-\tilde{\lambda}_{p}} \\
& =\sum_{p>1} \frac{\left(\nabla_{k} g_{1 \bar{p}}+\Gamma_{k 1}^{q} B_{q}^{p}\right)\left(\nabla_{\bar{k}} g_{p \overline{1}}\right)+\left(\nabla_{k} g_{p \overline{1}}-\Gamma_{k q}^{1} B_{p}^{q}\right)\left(\nabla_{\bar{k}} g_{1 \bar{p}}\right)}{\lambda_{1}-\tilde{\lambda}_{p}} \\
& \geqslant \frac{1}{2} \sum_{p>1} \frac{\left|\nabla_{k} g_{1 \bar{p}}\right|^{2}+\left|\nabla_{k} g_{p \overline{1}}\right|^{2}}{\lambda_{1}-\tilde{\lambda}_{p}}-C,
\end{aligned}
$$


where we used (3.3) and (3.6). Recall that, due to our choice of $B$, we have $\sum_{i} \tilde{\lambda}_{i}>-1$, which implies $1 /\left(\lambda_{1}-\tilde{\lambda}_{p}\right) \geqslant 1 /\left(n \lambda_{1}+1\right)$ for $p>1$, and so

$$
\tilde{\lambda}_{1}^{p q, r s}\left(\nabla_{k} \tilde{A}_{q}^{p}\right)\left(\nabla_{\bar{k}} \tilde{A}_{s}^{r}\right) \geqslant \frac{1}{2\left(n \lambda_{1}+1\right)} \sum_{p>1}\left(\left|\nabla_{k} g_{1 \bar{p}}\right|^{2}+\left|\nabla_{k} g_{p \overline{1}}\right|^{2}\right)-C .
$$

To rewrite this in terms of partial derivatives, note that

$$
\nabla_{k} g_{1 \bar{p}}=g_{1 \bar{p} k}-\Gamma_{k 1}^{\ell} g_{\ell \bar{p}}=g_{1 \bar{p} k}+O\left(\lambda_{1}\right),
$$

where we made use of the fact that $\sum_{i} \lambda_{i}>0$ to conclude that $\left|\lambda_{i}\right| \leqslant(n-1) \lambda_{1}$ for all $i$. It follows, since we assume $\lambda_{1}>1$, that

$$
\tilde{\lambda}_{1}^{p q, r s}\left(\nabla_{k} \tilde{A}_{q}^{p}\right)\left(\nabla_{\bar{k}} \tilde{A}_{s}^{r}\right) \geqslant \frac{1}{4 n \lambda_{1}} \sum_{p>1}\left(\left|g_{1 \bar{p} k}\right|^{2}+\left|g_{p \overline{1} k}\right|^{2}\right)-C \lambda_{1} .
$$

Combining this with (3.8) and (3.9), we obtain

$$
\begin{aligned}
\tilde{\lambda}_{1, k \bar{k}} & \geqslant g_{1 \overline{1} k \bar{k}}+\frac{1}{4 n \lambda_{1}} \sum_{p>1}\left(\left|g_{1 \bar{p} k}\right|^{2}+\left|g_{p \overline{1} k}\right|^{2}\right)-C\left(\sum_{m}\left|g_{1 \bar{m} k}\right|+\lambda_{1}\right) \\
& \geqslant g_{1 \overline{1} k \bar{k}}+\frac{1}{8 n \lambda_{1}} \sum_{p>1}\left(\left|g_{1 \bar{p} k}\right|^{2}+\left|g_{p \overline{1} k}\right|^{2}\right)-C\left(\left|g_{1 \overline{1} k}\right|+\lambda_{1}\right) .
\end{aligned}
$$

Rewriting $g$ in terms of $u$, we have

$$
\begin{aligned}
g_{1 \overline{1} k \bar{k}} & =\chi_{1 \overline{1} k \bar{k}}+u_{1 \overline{1} k \bar{k}}+W_{1 \overline{1} k \bar{k}}=\chi_{1 \overline{1} k \bar{k}}+u_{k \bar{k} 1 \overline{1}}+W_{1 \overline{1} k \bar{k}} \\
& =\chi_{1 \overline{1} k \bar{k}}-\chi_{k \bar{k} 1 \overline{1}}+g_{k \bar{k} \overline{1}}-W_{k \bar{k} 1 \overline{1}}+W_{1 \overline{1} k \bar{k}},
\end{aligned}
$$

and so

$$
\begin{aligned}
F^{k k} \tilde{\lambda}_{1, k \bar{k}} \geqslant & F^{k k} g_{k \bar{k} 1 \overline{1}}+F^{k k}\left(W_{1 \overline{1} k \bar{k}}-W_{k \bar{k} 1 \overline{1}}\right) \\
& +\frac{1}{8 n \lambda_{1}} \sum_{p>1} F^{k k}\left(\left|g_{1 \bar{p} k}\right|^{2}+\left|g_{p \overline{1} k}\right|^{2}\right)-C\left(F^{k k}\left|g_{1 \overline{1} k}\right|+\lambda_{1} \mathcal{F}\right),
\end{aligned}
$$

where $F^{p q}$ denotes the partial derivative of the function $F(A)$ with respect to the $(p, q)$ entry of the matrix $A$ (as explained earlier), and we have set $\mathcal{F}=\sum_{k} F^{k k}$. Observe that, due to (2.7) and (3.6), at the origin we have that $F^{p q}$ vanishes whenever $p \neq q$, while on the other hand $F^{k k}=f_{k}$, using the notation from $\S 2$. Recall from the last assertion of Proposition 2.3 that

$$
\mathcal{F} \geqslant \varkappa>0,
$$

for a uniform $\varkappa>0$. 


\section{- The term $F^{k k} g_{k \bar{k} 1 \overline{1}}$}

We now differentiate the equation $F(A)=h$, using covariant derivatives to simplify a term that appears below. Applying $\nabla_{i}$, we obtain

$$
F^{p q} \nabla_{i} g_{q \bar{p}}=h_{i}
$$

namely,

$$
F^{k k} g_{k \bar{k} i}+F^{k k}\left(\alpha^{k \bar{k}}\right)_{i} g_{k \bar{k}}=h_{i} .
$$

Applying $\nabla_{\bar{i}}$ and setting $i=1$,

$$
F^{p q, r s} \nabla_{1} g_{q \bar{p}} \nabla_{\overline{1}} g_{s \bar{r}}+F^{k k} \nabla_{\overline{1}} \nabla_{1} g_{k \bar{k}}=h_{1 \overline{1}} .
$$

To rewrite this using partial derivatives, note that

$$
\begin{aligned}
\nabla_{\overline{1}} \nabla_{1} g_{k \bar{k}} & =g_{k \bar{k} 1 \overline{1}}-\left(\partial_{\overline{1}} \Gamma_{1 k}^{m}\right) g_{m \bar{k}}-\Gamma_{1 k}^{m} g_{m \bar{k} \overline{1}}-\bar{\Gamma}_{1 k}^{q} g_{k \bar{q} 1}+\bar{\Gamma}_{1 k}^{q} \Gamma_{1 k}^{m} g_{m \bar{q}} \\
& =g_{k \bar{k} 1 \overline{1}}-2 \operatorname{Re}\left(\overline{\Gamma_{1 k}^{q}} g_{k \bar{q} 1}\right)+O\left(\lambda_{1}\right) .
\end{aligned}
$$

By rewriting $g$ in terms of $u$, we have

$$
g_{k \bar{q} 1}=g_{1 \bar{q} k}+\chi_{k \bar{q} 1}-\chi_{1 \bar{q} k}+W_{k \bar{q} 1}-W_{1 \bar{q} k}
$$

and hence

$$
\nabla_{\overline{1}} \nabla_{1} g_{k \bar{k}}=g_{k \bar{k} 1 \overline{1}}-2 \operatorname{Re}\left(\overline{\Gamma_{1 k}^{q}}\left(W_{k \bar{q} 1}-W_{1 \bar{q} k}\right)\right)+O\left(\sum_{q}\left|g_{1 \bar{q} k}\right|+\lambda_{1}\right) .
$$

Returning to (3.16), and making use of (3.14), we obtain

$$
\begin{gathered}
F^{k k} g_{k \bar{k} 1 \overline{1}} \geqslant-F^{p q, r s} \nabla_{1} g_{q \bar{p}} \nabla_{\overline{1}} g_{s \bar{r}}-C F^{k k} \sum_{q}\left|g_{1 \bar{q} k}\right|-C \mathcal{F} \lambda_{1} \\
+2 \operatorname{Re}\left(F^{k k} \overline{\Gamma_{1 k}^{q}}\left(W_{k \bar{q} 1}-W_{1 \bar{q} k}\right)\right) .
\end{gathered}
$$

To bound the term involving $W_{1 \bar{q} k}$, we observe that

$$
\left|F^{k k} W_{1 \bar{q} k}\right| \leqslant C\left(\mathcal{F} \lambda_{1}+\sum_{p} F^{k k}\left|u_{p k}\right|\right) .
$$

To see (3.19), we use the fact that $\lambda_{1}>K$ to bound the terms involving the gradient of $u$ that arise from $W_{1 \bar{q} k}$ when taking the $\partial / \partial z^{k}$ derivative of $W_{1 \bar{q}}=\left(\operatorname{tr}_{\alpha} Z\right) \alpha_{1 \bar{q}}-(n-1) Z_{1 \bar{q}}$. For the term involving $W_{k \bar{q} 1}$, note that

$$
\begin{aligned}
\nabla_{\overline{1}} \nabla_{1} W_{k \bar{k}} & =\nabla_{\overline{1}}\left(W_{k \bar{k} 1}-\Gamma_{1 k}^{q} W_{q \bar{k}}\right) \\
& =W_{k \bar{k} 1 \overline{1}}-\overline{\Gamma_{1 k}^{q}} W_{k \bar{q} 1}-\Gamma_{1 k}^{q} W_{q \bar{k} \overline{1}}-\left(\Gamma_{1 k}^{q}\right)_{\overline{1}} W_{q \bar{k}}+\bar{\Gamma}_{1 k}^{p} \Gamma_{1 k}^{q} W_{q \bar{p}} .
\end{aligned}
$$


In particular,

$$
2 \operatorname{Re}\left(\bar{\Gamma}_{1 k}^{q} W_{k \bar{q} 1}\right)=W_{k \bar{k} 1 \overline{1}}-\nabla_{\overline{1}} \nabla_{1} W_{k \bar{k}}+O\left(K^{1 / 2}\right) .
$$

Using this (and that we may assume $\lambda_{1}>K$ ), we have

$$
2 F^{k k} \operatorname{Re}\left(\bar{\Gamma}_{1 k}^{q} W_{k \bar{q} 1}\right)=F^{k k} W_{k \bar{k} 1 \overline{1}}-F^{k k} \nabla_{\overline{1}} \nabla_{1} W_{k \bar{k}}+O\left(\mathcal{F} \lambda_{1}\right)
$$

Combining (3.18)-(3.20) gives

$$
\begin{gathered}
F^{k k} g_{k \bar{k} 1 \overline{1}} \geqslant-F^{p q, r s} \nabla_{1} g_{q \bar{p}} \nabla_{\overline{1}} g_{s \bar{r}}+F^{k k} W_{k \bar{k} 1 \overline{1}}-F^{k k} \nabla_{\overline{1}} \nabla_{1} W_{k \bar{k}} \\
-C\left(F^{k k} \sum_{q}\left|g_{1 \bar{q} k}\right|+F^{k k} \sum_{p}\left|u_{p k}\right|+\mathcal{F} \lambda_{1}\right) .
\end{gathered}
$$

Going back to (3.13), using the square terms there to control the terms in (3.21) involving $\left|g_{1 \bar{q} k}\right|$ for $q \neq 1$, we obtain

$$
\begin{gathered}
F^{k k} \tilde{\lambda}_{1, k \bar{k}} \geqslant-F^{p q, r s} \nabla_{1} g_{q \bar{p}} \nabla_{\overline{1}} g_{s \bar{r}}+F^{k k}\left(W_{1 \overline{1} k \bar{k}}-\nabla_{\overline{1}} \nabla_{1} W_{k \bar{k}}\right) \\
-C\left(F^{k k}\left|g_{1 \overline{1} k}\right|+\sum_{p} F^{k k}\left|u_{p k}\right|+\lambda_{1} \mathcal{F}\right) .
\end{gathered}
$$

\section{- The term $F^{k k} \nabla_{\overline{1}} \nabla_{1} W_{k \bar{k}}$}

Using (2.5) and (2.11), we have

$$
\begin{aligned}
F^{k k} \nabla_{\overline{1}} \nabla_{1} W_{k \bar{k}} & =\frac{1}{n-1} \sum_{k} \sum_{i \neq k} \widetilde{F}^{i i} \nabla_{\overline{1}} \nabla_{1} W_{k \bar{k}} \\
& =\frac{1}{n-1} \sum_{i} \widetilde{F}^{i i} \sum_{k \neq i} \nabla_{\overline{1}} \nabla_{1} W_{k \bar{k}}=\widetilde{F}^{i i} \nabla_{\overline{1}} \nabla_{1} Z_{i \bar{i}}
\end{aligned}
$$

Recall from (2.12) and (2.13) that $\widetilde{F}^{11}=\tilde{f}_{1}$ is "large", equivalent to $F^{k k}=f_{k}$ for any $k>1$, while $\widetilde{F}^{i i}=\tilde{f}_{i}$ for $i>1$, is "small", bounded by $F^{11}=f_{1}$. We also recall that, as explained earlier, the crucial assumption on $Z_{i \bar{j}}$ implies that $\nabla_{\overline{1}} \nabla_{1} Z_{1 \overline{1}}$ does not contain the terms $u_{11 \overline{1}}, u_{11}$ or their complex conjugates.

Hence, using the fact that $\sup _{i, j}\left|u_{i \bar{j}}\right| \leqslant C \lambda_{1}$ and $\lambda_{1} \geqslant K$,

$$
\begin{aligned}
F^{k k} \nabla_{\overline{1}} \nabla_{1} W_{k \bar{k}} & \leqslant C\left(\widetilde{F}^{11} \sum_{k>1}\left(\left|u_{k 1}\right|+\left|u_{k \overline{1} 1}\right|\right)+F^{11} \sum_{k}\left(\left|u_{k 1}\right|+\left|u_{k \overline{1} 1}\right|\right)+\lambda_{1} \mathcal{F}\right) \\
& \leqslant C\left(F^{11}\left(\left|u_{11}\right|+\left|u_{1 \overline{1} 1}\right|\right)+\sum_{k>1} F^{k k}\left(\left|u_{k 1}\right|+\left|u_{k \overline{1} 1}\right|\right)+\lambda_{1} \mathcal{F}\right) \\
& \leqslant C\left(\sum_{p} F^{k k}\left|u_{p k}\right|+F^{k k}\left|u_{1 \overline{1} k}\right|+\lambda_{1} \mathcal{F}\right) .
\end{aligned}
$$


We also have

$$
u_{1 \overline{1} k}=g_{1 \overline{1} k}-\chi_{1 \overline{1} k}-W_{1 \overline{1} k},
$$

and so

$$
F^{k k}\left|u_{1 \overline{1} k}\right| \leqslant F^{k k}\left|g_{1 \overline{1} k}\right|+C\left(\sum_{p} F^{k k}\left|u_{p k}\right|+\lambda_{1} \mathcal{F}\right) .
$$

From (3.24), we then obtain

$$
F^{k k} \nabla_{\overline{1}} \nabla_{1} W_{k \bar{k}} \leqslant C\left(\sum_{p} F^{k k}\left|u_{p k}\right|+F^{k k}\left|g_{1 \overline{1} k}\right|+\lambda_{1} \mathcal{F}\right) .
$$

\section{- The term $F^{k k} W_{1 \overline{1} k \bar{k}}$}

Let us write $W_{1 \overline{1}}=W^{p} u_{p}+W^{\bar{p}} u_{\bar{p}}$. We have

$$
\left(W^{p} u_{p}\right)_{k \bar{k}}=\left(W^{p}\right)_{k \bar{k}} u_{p}+\left(W^{p}\right)_{k} u_{p \bar{k}}+\left(W^{p}\right)_{\bar{k}} u_{p k}+W^{p} u_{p k \bar{k}}
$$

and so, since we may assume that $\lambda_{1} \gg K$, it follows that

$$
F^{k k}\left(W^{p} u_{p}\right)_{k \bar{k}} \geqslant W^{p} F^{k k} u_{k \bar{k} p}-C\left(\sum_{p} F^{k k}\left|u_{p k}\right|+\lambda_{1} \mathcal{F}\right) .
$$

Using (3.15), we have

$$
\begin{aligned}
\left|F^{k k} u_{k \bar{k} p}\right|=\left|F^{k k}\left(g_{k \bar{k} p}-\chi_{k \bar{k} p}-W_{k \bar{k} p}\right)\right| & \leqslant C F^{k k}\left|g_{k \bar{k}}\right|+C \mathcal{F}+\left|F^{k k} W_{k \bar{k} p}\right| \\
& \leqslant C F^{k k}\left|u_{k \bar{k}}\right|+C K^{1 / 2} \mathcal{F}+\left|F^{k k} W_{k \bar{k} p}\right| .
\end{aligned}
$$

To deal with this last term, note that, due to (2.5), as in (3.23) we have

$$
F^{k k} W_{k \bar{k} p}=F^{k k} \nabla_{p} W_{k \bar{k}}+O\left(K^{1 / 2} \mathcal{F}\right)=\widetilde{F}^{i i} \nabla_{p} Z_{i \bar{i}}+O\left(K^{1 / 2} \mathcal{F}\right),
$$

and using the crucial assumption on $Z_{i \bar{j}}$, as explained earlier, we see that $\nabla_{p} Z_{1 \overline{1}}$ is independent of $u_{11}$ and $u_{1 \overline{1}}$. It follows that these Hessian terms can appear only with the "small" coefficients $\widetilde{F}^{i i}$ with $i>1$. We obtain

$$
\begin{aligned}
\left|F^{k k} W_{k \bar{k} p}\right| & \leqslant C\left(\widetilde{F}^{11} \sum_{k>1}\left(\left|u_{k p}\right|+\left|u_{\bar{k} p}\right|\right)+F^{11} \sum_{k}\left(\left|u_{k p}\right|+\left|u_{\bar{k} p}\right|\right)+K^{1 / 2} \mathcal{F}\right) \\
& \leqslant C\left(\sum_{p} F^{k k}\left|u_{p k}\right|+\sum_{p} F^{k k}\left|u_{p \bar{k}}\right|+K^{1 / 2} \mathcal{F}\right),
\end{aligned}
$$

and so

$$
\left|F^{k k} u_{k \bar{k} p}\right| \leqslant C\left(\sum_{p} F^{k k}\left|u_{p k}\right|+\sum_{p} F^{k k}\left|u_{p \bar{k}}\right|+K^{1 / 2} \mathcal{F}\right) .
$$


From (3.26), we then have (using $\lambda_{1} \gg K$ )

$$
F^{k k}\left(W^{p} u_{p}\right)_{k \bar{k}} \geqslant-C\left(\sum_{p} F^{k k}\left|u_{p k}\right|+\sum_{p} F^{k k}\left|u_{p \bar{k}}\right|+\lambda_{1} \mathcal{F}\right) .
$$

A similar argument gives the same estimate for $F^{k k}\left(W^{\bar{p}} u_{\bar{p}}\right)_{k \bar{k}}$, and this completes the required estimate for $F^{k k} W_{1 \overline{1} k \bar{k}}$ :

$$
F^{k k} W_{1 \overline{1} k \bar{k}} \geqslant-C\left(\sum_{p} F^{k k}\left|u_{p k}\right|+\lambda_{1} \mathcal{F}\right) .
$$

Putting together this last inequality and (3.25) into (3.22), we obtain

$$
F^{k k} \tilde{\lambda}_{1, k \bar{k}} \geqslant-F^{p q, r s} \nabla_{1} g_{q \bar{p}} \nabla_{\overline{1}} g_{s \bar{r}}-C\left(F^{k k}\left|g_{1 \overline{1} k}\right|+\sum_{p} F^{k k}\left|u_{p k}\right|+\lambda_{1} \mathcal{F}\right) .
$$

We now use this in equation (3.5) to give

$$
\begin{aligned}
F^{k k} \widetilde{H}_{k \bar{k}} \geqslant- & \frac{F^{p q, r s} \nabla_{1} g_{q \bar{p}} \nabla_{\overline{1}} g_{s \bar{r}}}{\lambda_{1}}-\frac{F^{k k}\left|\tilde{\lambda}_{1, k}\right|^{2}}{\lambda_{1}^{2}}+F^{k k} \phi^{\prime}\left(u_{p} u_{\bar{p} k \bar{k}}+u_{\bar{p}} u_{p k \bar{k}}\right) \\
& +\sum_{p} \frac{F^{k k}}{5 K}\left(\left|u_{p k}\right|^{2}+\left|u_{\bar{p} k}\right|^{2}\right)+\phi^{\prime \prime} F^{k k}\left|V_{k}\right|^{2}+\psi^{\prime \prime} F^{k k}\left|u_{k}\right|^{2}+\psi^{\prime} F^{k k} u_{k \bar{k}} \\
& -C\left(F^{k k} \lambda_{1}^{-1}\left|g_{1 \overline{1} k}\right|+\sum_{p} F^{k k} \lambda_{1}^{-1}\left|u_{p k}\right|+\mathcal{F}\right) .
\end{aligned}
$$

We can use (3.27) and the fact that $\phi^{\prime}<1 / 2 K$ to bound the terms involving $u_{\bar{p} k \bar{k}}$ and $u_{p k \bar{k}}$ :

$$
\sum_{p} \phi^{\prime} F^{k k}\left|u_{p} u_{\bar{p} k \bar{k}}\right| \leqslant C\left(\frac{1}{K^{1 / 2}} \sum_{p} F^{k k}\left|u_{p k}\right|+\frac{1}{K^{1 / 2}} \sum_{p} F^{k k}\left|u_{p \bar{k}}\right|+\mathcal{F}\right),
$$

which in turn can be controlled by the good squared terms $\left|u_{p k}\right|^{2}+\left|u_{p \bar{k}}\right|^{2}$ at the cost of an extra multiple of $\mathcal{F}$. In addition, since we assume $\lambda_{1} \gg K$, we can control the $F^{k k} \lambda_{1}^{-1}\left|u_{p k}\right|$ term in the same way. We therefore have

$$
\begin{aligned}
0 \geqslant F^{k k} \widetilde{H}_{k \bar{k}} \geqslant- & \frac{F^{p q, r s} \nabla_{1} g_{q \bar{p}} \nabla_{\overline{1}} g_{s \bar{r}}}{\lambda_{1}}-\frac{F^{k k}\left|\tilde{\lambda}_{1, k}\right|^{2}}{\lambda_{1}^{2}} \\
& +\sum_{p} \frac{F^{k k}}{6 K}\left(\left|u_{p k}\right|^{2}+\left|u_{p \bar{k}}\right|^{2}\right)+\phi^{\prime \prime} F^{k k}\left|V_{k}\right|^{2} \\
& +\psi^{\prime \prime} F^{k k}\left|u_{k}\right|^{2}+\psi^{\prime} F^{k k} u_{k \bar{k}}-C\left(F^{k k} \lambda_{1}^{-1}\left|g_{1 \overline{1} k}\right|+\mathcal{F}\right) .
\end{aligned}
$$


We now deal with two cases separately, as was done in Hou-Ma-Wu [20], depending on a small constant $\delta=\delta_{D_{1}, D_{2}}>0$ to be determined shortly, and which will depend on the constants $D_{1}$ and $D_{2}$.

Case 1. Assume $\delta \lambda_{1} \geqslant-\lambda_{n}$. Define the set

$$
I=\left\{i: F^{i i}>\delta^{-1} F^{11}\right\} .
$$

From (3.4) and the fact that $\widetilde{H}_{k}=0$ at the maximum, we get

$$
\begin{aligned}
-\sum_{k \notin I} \frac{F^{k k}\left|\tilde{\lambda}_{1, k}\right|^{2}}{\lambda_{1}^{2}} & =-\sum_{k \notin I} F^{k k}\left|\phi^{\prime} V_{k}+\psi^{\prime} u_{k}\right|^{2} \\
& \geqslant-2\left(\phi^{\prime}\right)^{2} \sum_{k \notin I} F^{k k}\left|V_{k}\right|^{2}-2\left(\psi^{\prime}\right)^{2} \sum_{k \notin I} F^{k k}\left|u_{k}\right|^{2} \\
& \geqslant-\phi^{\prime \prime} \sum_{k \notin I} F^{k k}\left|V_{k}\right|^{2}-2\left(\psi^{\prime}\right)^{2} \delta^{-1} F^{11} K .
\end{aligned}
$$

For $k \in I$ we have, in the same way,

$$
-2 \delta \sum_{k \in I} \frac{F^{k k}\left|\tilde{\lambda}_{1, k}\right|^{2}}{\lambda_{1}^{2}} \geqslant-2 \delta \phi^{\prime \prime} \sum_{k \in I} F^{k k}\left|V_{k}\right|^{2}-4 \delta\left(\psi^{\prime}\right)^{2} \sum_{k \in I} F^{k k}\left|u_{k}\right|^{2} .
$$

We wish to use some of the good $\psi^{\prime \prime} F^{k k}\left|u_{k}\right|^{2}$ term in (3.28) to control the last term in (3.30). For this, we assume that $\delta$ is chosen so small (depending on $\psi$, i.e. on $D_{1}, D_{2}$ and the maximum of $|u|)$, such that

$$
4 \delta\left(\psi^{\prime}\right)^{2}<\frac{1}{2} \psi^{\prime \prime}
$$

Since $\psi^{\prime \prime}$ is strictly positive, such a $\delta>0$ exists.

Using this together with (3.29) and (3.30) in (3.28), we have

$$
\begin{aligned}
0 \geqslant- & \frac{F^{p q, r s} \nabla_{1} g_{q \bar{p}} \nabla_{\overline{1}} g_{s \bar{r}}}{\lambda_{1}}-(1-2 \delta) \sum_{k \in I} \frac{F^{k k}\left|\tilde{\lambda}_{1, k}\right|^{2}}{\lambda_{1}^{2}} \\
& +\sum_{p} \frac{F^{k k}}{6 K}\left(\left|u_{p k}\right|^{2}+\left|u_{p \bar{k}}\right|^{2}\right)+\frac{1}{2} \psi^{\prime \prime} F^{k k}\left|u_{k}\right|^{2}+\psi^{\prime} F^{k k} u_{k \bar{k}} \\
& -2\left(\psi^{\prime}\right)^{2} \delta^{-1} F^{11} K-C\left(F^{k k} \lambda_{1}^{-1}\left|g_{1 \overline{1} k}\right|+\mathcal{F}\right) .
\end{aligned}
$$

To deal with the first two terms, note that (as in [34, equation (67)]) the concavity of the operator $F$ implies that

$$
-F^{p q, r s} \nabla_{1} g_{q \bar{p}} \nabla_{\overline{1}} g_{s \bar{r}} \geqslant \sum_{k \in I} \frac{F^{k k}-F^{11}}{\lambda_{1}-\lambda_{k}}\left|\nabla_{1} g_{k \overline{1}}\right|^{2},
$$


where note that the denominator involves $\lambda_{k}$ instead of $\tilde{\lambda}_{k}$ because we are evaluating $F$ at $A$. We also remark that the denominator on the right-hand side does not vanish, because the assumption $k \in I$ implies that $F^{k k}>F^{11}$, which in turn implies that $\lambda_{k}<\lambda_{1}$ because $f$ is symmetric. By definition, for $k \in I$ we have $F^{11} \leqslant \delta F^{k k}$, and the assumption $\delta \lambda_{1} \geqslant-\lambda_{n}$ implies that

$$
\frac{1-\delta}{\lambda_{1}-\lambda_{k}} \geqslant \frac{1-2 \delta}{\lambda_{1}}
$$

It follows that

$$
\sum_{k \in I} \frac{F^{k k}-F^{11}}{\lambda_{1}-\lambda_{k}}\left|\nabla_{1} g_{k \overline{1}}\right|^{2} \geqslant \sum_{k \in I} \frac{(1-\delta) F^{k k}}{\lambda_{1}-\lambda_{k}}\left|\nabla_{1} g_{k \overline{1}}\right|^{2} \geqslant \frac{1-2 \delta}{\lambda_{1}} \sum_{k \in I} F^{k k}\left|\nabla_{1} g_{k \overline{1}}\right|^{2} .
$$

Combining this with (3.32) and (3.33), we then obtain

$$
\begin{aligned}
0 \geqslant(1 & -2 \delta) \sum_{k \in I} \frac{F^{k k}\left(\left|\nabla_{1} g_{k \overline{1}}\right|^{2}-\left|\tilde{\lambda}_{1, k}\right|^{2}\right)}{\lambda_{1}^{2}} \\
& +\sum_{p} \frac{F^{k k}}{6 K}\left(\left|u_{p k}\right|^{2}+\left|u_{p \bar{k}}\right|^{2}\right)+\frac{1}{2} \psi^{\prime \prime} F^{k k}\left|u_{k}\right|^{2}+\psi^{\prime} F^{k k} u_{k \bar{k}} \\
& -2\left(\psi^{\prime}\right)^{2} \delta^{-1} F^{11} K-C\left(F^{k k} \lambda_{1}^{-1}\left|g_{1 \overline{1} k}\right|+\mathcal{F}\right)
\end{aligned}
$$

We wish to obtain a lower bound for the first term in (3.35). We make the following claim.

Claim. For any $\varepsilon>0$ there exists a constant $C_{\varepsilon}$ such that

$$
\begin{gathered}
\sum_{k \in I} \frac{F^{k k}\left|\nabla_{1} g_{k \overline{1}}\right|^{2}}{\lambda_{1}^{2}} \geqslant \sum_{k \in I} \frac{F^{k k}\left|\tilde{\lambda}_{1, k}\right|^{2}}{\lambda_{1}^{2}}-\sum_{p} \frac{F^{k k}}{12 K}\left(\left|u_{p \bar{k}}\right|^{2}+\left|u_{p k}\right|^{2}\right) \\
+C_{\varepsilon} \psi^{\prime} F^{k k}\left|u_{k}\right|^{2}+\varepsilon C \psi^{\prime} \mathcal{F}-C \mathcal{F}
\end{gathered}
$$

as long as $\lambda_{1} / K$ is sufficiently large compared to $\psi^{\prime}$ (the constants $D_{1}$ and $D_{2}$ of $\psi$ will be chosen uniformly later).

Proof. First, we compare $\nabla_{1} g_{k \overline{1}}$ to $\tilde{\lambda}_{1, k}$. We have

$$
\begin{aligned}
\nabla_{1} g_{k \overline{1}}=g_{k \overline{1} 1}-\Gamma_{1 k}^{p} g_{p \overline{1}} & =\chi_{k \overline{1} 1}+u_{k \overline{1} 1}+W_{k \overline{1} 1}+O\left(\lambda_{1}\right)=\chi_{k \overline{1} 1}+u_{1 \overline{1} k}+W_{k \overline{1} 1}+O\left(\lambda_{1}\right) \\
& =g_{1 \overline{1} k}-W_{1 \overline{1} k}+W_{k \overline{1} 1}+O\left(\lambda_{1}\right)=\tilde{\lambda}_{1, k}-W_{1 \overline{1} k}+W_{k \overline{1} 1}+O\left(\lambda_{1}\right)
\end{aligned}
$$

absorbing bounded terms into $O\left(\lambda_{1}\right)$ and using (3.7). It follows that for any $k$, without summing,

$$
\left|\nabla_{1} g_{k \overline{1}}\right|^{2} \geqslant\left|\tilde{\lambda}_{1, k}\right|^{2}-C\left(\left|\tilde{\lambda}_{1, k}\right|\left(\left|W_{1 \overline{1} k}\right|+\left|W_{k \overline{1} 1}\right|\right)+\lambda_{1}\left|\tilde{\lambda}_{1, k}\right|+\left|W_{1 \overline{1} k}\right|^{2}+\left|W_{k \overline{1} 1}\right|^{2}+\lambda_{1}^{2}\right)
$$




\section{- The terms in (3.37) involving $\boldsymbol{W}$}

Note that if $k \in I$ then $k \neq 1$, and so from (2.5) we have

$$
W_{k \overline{1} 1}=\left(\operatorname{tr}_{\alpha} Z\right) \alpha_{k \overline{1} 1}-(n-1) Z_{k \overline{1} 1} .
$$

Our basic assumption for $Z$ implies that $Z_{k \overline{1} 1}=\nabla_{1} Z_{k \overline{1}}+O(Z)$ does not contain the Hessian terms $u_{11}$ and $u_{\overline{1} \overline{1}}$. It follows that $W_{k \overline{1} 1}$ and its complex conjugate do not contain these Hessian terms. The term $W_{1 \overline{1} k}$ and its complex conjugate also do not contain the Hessian terms $u_{11}$ and $u_{\overline{1} \overline{1}}$, since each Hessian term must contain a $k$-derivative. To simplify the formulas, let us write

$$
U=\sum_{\substack{p>1 \\ q \geqslant 1}}\left|u_{p q}\right|
$$

It follows that

$$
\left|W_{k \overline{1} 1}\right|+\left|W_{1 \overline{1} k}\right| \leqslant C\left(\lambda_{1}+U\right),
$$

and so the terms $\left|W_{k \overline{1} 1}\right|^{2}+\left|W_{1 \overline{1} k}\right|^{2}$ in (3.37) can be bounded by $C\left(\lambda_{1}^{2}+U^{2}\right)$.

We now use these to estimate the negative terms in (3.37). Using $\widetilde{H}_{k}=0$ together with (3.38), we have

$$
\begin{aligned}
& \left|\tilde{\lambda}_{1, k}\right|\left(\left|W_{1 \overline{1} k}\right|+\left|W_{k \overline{1} 1}\right|\right) \\
& \quad=\lambda_{1}\left|\phi^{\prime}\left(u_{r} u_{\bar{r} k}+u_{r k} u_{\bar{r}}+\left(\alpha^{r \bar{s}}\right)_{k} u_{r} u_{\bar{s}}\right)+\psi^{\prime} u_{k}\right|\left(\left|W_{1 \overline{1} k}\right|+\left|W_{k \overline{1} 1}\right|\right) \\
& \quad \leqslant \frac{C \lambda_{1}}{2 K^{1 / 2}}\left(\sum_{r}\left|u_{\bar{r} k}\right|+\sum_{r}\left|u_{r k}\right|+K^{1 / 2}\right)\left(\lambda_{1}+U\right)+C \lambda_{1}\left|\psi^{\prime}\right|\left|u_{k}\right|\left(\lambda_{1}+U\right) .
\end{aligned}
$$

We have

$$
\frac{C \lambda_{1}}{2 K^{1 / 2}}\left(\sum_{r}\left|u_{\bar{r} k}\right|+\sum_{r}\left|u_{r k}\right|+K^{1 / 2}\right) \lambda_{1} \leqslant \frac{C \lambda_{1}^{2}}{2 K^{1 / 2}} \sum_{r}\left|u_{\bar{r} k}\right|+\frac{C \lambda_{1}^{2}}{2 K^{1 / 2}} U+C \lambda_{1}^{2}
$$

and

$$
\begin{aligned}
\frac{C \lambda_{1}}{2 K^{1 / 2}}\left(\sum_{r}\left|u_{\bar{r} k}\right|+\sum_{r}\left|u_{r k}\right|+K^{1 / 2}\right) U & \leqslant \frac{C \lambda_{1}^{2}}{K^{1 / 2}} U+\frac{C \lambda_{1}}{K^{1 / 2}} U^{2}+C \lambda_{1} U \\
& \leqslant \frac{C \lambda_{1}^{2}}{K^{1 / 2}} U+\frac{C \lambda_{1}}{K^{1 / 2}} U^{2} .
\end{aligned}
$$

Next, for any $\varepsilon>0$, there exists a constant $C_{\varepsilon}$ such that

$$
\lambda_{1}\left|\psi^{\prime}\right|\left|u_{k}\right| \lambda_{1} \leqslant-\varepsilon \lambda_{1}^{2} \psi^{\prime}-\lambda_{1}^{2} C_{\varepsilon} \psi^{\prime}\left|u_{k}\right|^{2},
$$


where we have used the fact that $\psi^{\prime}<0$. Also,

$$
\lambda_{1}\left|\psi^{\prime}\right|\left|u_{k}\right| U \leqslant-\lambda_{1} \psi^{\prime} U^{2}-\lambda_{1} \psi^{\prime}\left|u_{k}\right|^{2}
$$

Combining (3.39) with (3.40)-(3.43), we obtain

$$
\begin{aligned}
\left|\tilde{\lambda}_{1, k}\right|\left(\left|W_{1 \overline{1} k}\right|+\left|W_{k \overline{1} 1}\right|\right) \leqslant C( & \frac{\lambda_{1}^{2}}{2 K^{1 / 2}} \sum_{r}\left|u_{\bar{r} k}\right|+\frac{\lambda_{1}^{2}}{K^{1 / 2}} U+\frac{\lambda_{1}}{K^{1 / 2}} U^{2}+\lambda_{1}^{2} \\
& \left.\quad-\lambda_{1}^{2} C_{\varepsilon} \psi^{\prime}\left|u_{k}\right|^{2}-\varepsilon \lambda_{1}^{2} \psi^{\prime}-\lambda_{1} \psi^{\prime}\left|u_{k}\right|^{2}-\lambda_{1} \psi^{\prime} U^{2}\right) .
\end{aligned}
$$

Using $\widetilde{H}_{k}=0$ again,

$$
\begin{aligned}
\frac{\left|\tilde{\lambda}_{1, k}\right|}{\lambda_{1}} & =\left|\phi^{\prime}\left(u_{p} u_{\bar{p} k}+u_{p k} u_{\bar{p}}+\left(\alpha^{p \bar{q}}\right)_{k} u_{p} u_{\bar{q}}\right)+\psi^{\prime} u_{k}\right| \\
& \leqslant \frac{1}{2 K^{1 / 2}} \sum_{p}\left|u_{\bar{p} k}\right|+\frac{1}{2 K^{1 / 2}} U-C_{\varepsilon} \psi^{\prime}\left|u_{k}\right|^{2}-\varepsilon \psi^{\prime}+C .
\end{aligned}
$$

We then obtain

$$
\begin{aligned}
\frac{\left|\nabla_{1} g_{k \overline{1}}\right|^{2}}{\lambda_{1}^{2}} \geqslant \frac{\left|\tilde{\lambda}_{1, k}\right|^{2}}{\lambda_{1}^{2}}-C\left(\frac{1}{K^{1 / 2}} \sum_{p}\left|u_{p \bar{k}}\right|+\frac{1}{K^{1 / 2}} U\right. \\
\left.\quad+\frac{1}{\lambda_{1} K^{1 / 2}} U^{2}+1-\varepsilon \psi^{\prime}-\frac{1}{\lambda_{1}} \psi^{\prime}\left|u_{k}\right|^{2}-\frac{1}{\lambda_{1}} \psi^{\prime} U^{2}\right)+C_{\varepsilon} \psi^{\prime}\left|u_{k}\right|^{2} .
\end{aligned}
$$

Summing over $k \in I$, we have

$$
\begin{aligned}
\sum_{k \in I} \frac{F^{k k}\left|\nabla_{1} g_{k}\right|^{2}}{\lambda_{1}^{2}} \geqslant \sum_{k \in I} & \frac{F^{k k}\left|\tilde{\lambda}_{1, k}\right|^{2}}{\lambda_{1}^{2}}-C\left(\frac{1}{K^{1 / 2}} \sum_{p} F^{k k}\left|u_{p \bar{k}}\right|+\frac{1}{K^{1 / 2}} \mathcal{F} U\right. \\
& \left.+\frac{1}{\lambda_{1} K^{1 / 2}} \mathcal{F} U^{2}+\mathcal{F}-\varepsilon \mathcal{F} \psi^{\prime}-\frac{1}{\lambda_{1}} \psi^{\prime} F^{k k}\left|u_{k}\right|^{2}-\frac{1}{\lambda_{1}} \psi^{\prime} \mathcal{F} U^{2}\right) \\
& +C_{\varepsilon} \psi^{\prime} F^{k k}\left|u_{k}\right|^{2} .
\end{aligned}
$$

First, we use

$$
\frac{C}{K^{1 / 2}} \sum_{p} F^{k k}\left|u_{p \bar{k}}\right| \leqslant \frac{1}{12 K} \sum_{p} F^{k k}\left|u_{p \bar{k}}\right|^{2}+C \mathcal{F} .
$$

Note that all $F^{k k}$, with $k>1$, are comparable to $\mathcal{F}$. It follows that

$$
\frac{C}{K^{1 / 2}} \mathcal{F} U \leqslant \frac{1}{50 K} \sum_{p} F^{k k}\left|u_{p k}\right|^{2}+C \mathcal{F}
$$

and

$$
\frac{C}{\lambda_{1} K^{1 / 2}} \mathcal{F} U^{2} \leqslant \frac{C}{\lambda_{1} K^{1 / 2}} \sum_{p} F^{k k}\left|u_{p k}\right|^{2} \leqslant \frac{1}{50 K} \sum_{p} F^{k k}\left|u_{p k}\right|^{2} .
$$


As long as $\lambda_{1} / K$ is sufficiently large depending on $\psi^{\prime}$ (i.e. depending on $D_{1}$ and $D_{2}$, which will be chosen later), we have

$$
\frac{C}{\lambda_{1}} \psi^{\prime} \mathcal{F} U^{2} \leqslant \frac{1}{50 K} \sum_{p} F^{k k}\left|u_{p k}\right|^{2} .
$$

and using (3.47)-(3.50) in (3.46), we finally obtain

$$
\begin{gathered}
\sum_{k \in I} \frac{F^{k k}\left|\nabla_{1} g_{k \overline{1}}\right|^{2}}{\lambda_{1}^{2}} \geqslant \sum_{k \in I} \frac{F^{k k}\left|\tilde{\lambda}_{1, k}\right|^{2}}{\lambda_{1}^{2}}-\sum_{p} \frac{F^{k k}}{12 K}\left(\left|u_{p \bar{k}}\right|^{2}+\left|u_{p k}\right|^{2}\right) \\
+C_{\varepsilon} \psi^{\prime} F^{k k}\left|u_{k}\right|^{2}+\varepsilon C \psi^{\prime} \mathcal{F}-C \mathcal{F}
\end{gathered}
$$

This completes the proof of the claim.

We now use the claim in (3.35) to obtain

$$
\begin{aligned}
0 \geqslant \sum_{p} & \frac{F^{k k}}{12 K}\left(\left|u_{p k}\right|^{2}+\left|u_{p \bar{k}}\right|^{2}\right)+\frac{1}{2} \psi^{\prime \prime} F^{k k}\left|u_{k}\right|^{2}+\psi^{\prime} F^{k k} u_{k \bar{k}} \\
& \quad-2\left(\psi^{\prime}\right)^{2} \delta^{-1} F^{11} K-C\left(F^{k k} \lambda_{1}^{-1}\left|g_{1 \overline{1} k}\right|+\mathcal{F}\right)+C_{\varepsilon} \psi^{\prime} F^{k k}\left|u_{k}\right|^{2}+\varepsilon C \psi^{\prime} \mathcal{F} .
\end{aligned}
$$

\section{- The terms involving $\left|g_{1 \overline{1} k}\right|$ and $F^{k k} u_{k \bar{k}}$}

From (3.7) we know that

$$
g_{1 \overline{1} k}=\tilde{\lambda}_{1, k}+O\left(\lambda_{1}\right),
$$

and so using (3.45) we get

$$
F^{k k} \lambda_{1}^{-1}\left|g_{1 \overline{1} k}\right| \leqslant \frac{1}{2 K^{1 / 2}} \sum_{p} F^{k k}\left(\left|u_{\bar{p} k}\right|+\left|u_{p k}\right|\right)-C_{\varepsilon} \psi^{\prime} F^{k k}\left|u_{k}\right|^{2}-\varepsilon \psi^{\prime} \mathcal{F}+C \mathcal{F} .
$$

The terms involving $\left|u_{\bar{p} k}\right|$ and $\left|u_{p k}\right|$ can be absorbed by the squared terms $\left|u_{p \bar{k}}\right|^{2}$ and $\left|u_{p k}\right|^{2}$ in (3.51), and so we obtain

$$
\begin{array}{r}
0 \geqslant \sum_{p} \frac{F^{k k}}{20 K}\left(\left|u_{p k}\right|^{2}+\left|u_{p \bar{k}}\right|^{2}\right)+\frac{1}{2} \psi^{\prime \prime} F^{k k}\left|u_{k}\right|^{2}+\psi^{\prime} F^{k k} u_{k \bar{k}} \\
-2\left(\psi^{\prime}\right)^{2} \delta^{-1} F^{11} K-C \mathcal{F}+C_{\varepsilon} \psi^{\prime} F^{k k}\left|u_{k}\right|^{2}+\varepsilon C \psi^{\prime} \mathcal{F} .
\end{array}
$$

As for the term involving $u_{k \bar{k}}$, we have

$$
\psi^{\prime} F^{k k} u_{k \bar{k}}=\psi^{\prime} F^{k k}\left(g_{k \bar{k}}-\chi_{k \bar{k}}-W_{k \bar{k}}\right)
$$


As in (3.23), we have

$$
\sum_{k} F^{k k} W_{k \bar{k}}=\sum_{i} \widetilde{F}^{i i} Z_{i \bar{i}}
$$

Recall that $Z_{1 \overline{1}}$ does not contain $u_{1}$ and $u_{\overline{1}}$, and $\widetilde{F}^{11}$ is the only "large" coefficient of order $F^{k k}$ for $k>1$. It follows that

$$
\left|F^{k k} W_{k \bar{k}}\right| \leqslant C F^{k k}\left|u_{k}\right| \leqslant C_{\varepsilon} F^{k k}\left|u_{k}\right|^{2}+\varepsilon \mathcal{F}
$$

and so

$$
\psi^{\prime} F^{k k} u_{k \bar{k}} \geqslant \psi^{\prime} F^{k k}\left(g_{k \bar{k}}-\chi_{k \bar{k}}\right)+C_{\varepsilon} \psi^{\prime} F^{k k}\left|u_{k}\right|^{2}+\varepsilon \psi^{\prime} \mathcal{F}
$$

From (3.53), we then finally obtain (if necessary replacing $C_{\varepsilon}$ by another constant depending only on $\varepsilon$ and the allowed data), that

$$
\begin{aligned}
0 \geqslant F^{11} & \left(\frac{\lambda_{1}^{2}}{40 K}-2\left(\psi^{\prime}\right)^{2} \delta^{-1} K\right)+\left(\frac{1}{2} \psi^{\prime \prime}+C_{\varepsilon} \psi^{\prime}\right) F^{k k}\left|u_{k}\right|^{2} \\
& -C_{0} \mathcal{F}+\varepsilon C_{0} \psi^{\prime} \mathcal{F}-\psi^{\prime} F^{k k}\left(\chi_{k \bar{k}}-g_{k \bar{k}}\right),
\end{aligned}
$$

for a uniform $C_{0}$. We have used the fact that $\left|u_{1 \overline{1}}\right|^{2} \geqslant \frac{1}{2} \lambda_{1}^{2}-C K$.

Under the assumption that the function $\underline{u}=0$ is a $\mathcal{C}$-subsolution, and that $\lambda_{1} \gg 1$, we may apply Proposition 2.3 and see that there is a uniform positive number $\varkappa>0$ such that one of two possibilities occurs:

(a) We have $F^{k k}\left(\chi_{k \bar{k}}-g_{k \bar{k}}\right)>\varkappa \mathcal{F}$. In this case we have

$$
0 \geqslant F^{11}\left(\frac{\lambda_{1}^{2}}{40 K}-2\left(\psi^{\prime}\right)^{2} \delta^{-1} K\right)+\left(\frac{1}{2} \psi^{\prime \prime}+C_{\varepsilon} \psi^{\prime}\right) F^{k k}\left|u_{k}\right|^{2}-C_{0} \mathcal{F}+\varepsilon C_{0} \psi^{\prime} \mathcal{F}-\psi^{\prime} \varkappa \mathcal{F}
$$

We first choose $\varepsilon>0$ such that $\varepsilon C_{0}<\frac{1}{2} \varkappa$. We then choose the parameter $D_{2}$ in the definition of $\psi(t)=D_{1} e^{-D_{2} t}$ to be large enough so that

$$
\frac{1}{2} \psi^{\prime \prime}>C_{\varepsilon}\left|\psi^{\prime}\right|
$$

At this point, we have

$$
0 \geqslant F^{11}\left(\frac{\lambda_{1}^{2}}{40 K}-2\left(\psi^{\prime}\right)^{2} \delta^{-1} K\right)-C_{0} \mathcal{F}-\frac{1}{2} \psi^{\prime} \varkappa \mathcal{F}
$$

We now choose $D_{1}$ so large that $-\frac{1}{2} \psi^{\prime} \varkappa>C_{0}$, which implies

$$
\frac{\lambda_{1}^{2}}{40 K} \leqslant 2\left(\psi^{\prime}\right)^{2} \delta^{-1} K
$$


Note that $\delta$ is determined by the choices of $D_{1}$ and $D_{2}$, according to (3.31), so we obtain the required upper bound for $\lambda_{1} / K$.

(b) We have $F^{11}>\varkappa \mathcal{F}$. With the choices of constants made above, (3.55) implies that

$$
0 \geqslant \varkappa \mathcal{F}\left(\frac{\lambda_{1}^{2}}{40 K}-2\left(\psi^{\prime}\right)^{2} \delta^{-1} K\right)-C_{0} \mathcal{F}+\varepsilon C_{0} \psi^{\prime} \mathcal{F}+C_{1} \psi^{\prime} \mathcal{F}+\psi^{\prime} F^{k k} g_{k \bar{k}},
$$

for another uniform constant $C_{1}$. Since $F^{k k} g_{k \bar{k}} \leqslant \mathcal{F} \lambda_{1}$, we can divide through by $\mathcal{F} K$ and obtain

$$
0 \geqslant \frac{\varkappa \lambda_{1}^{2}}{40 K^{2}}-C_{2}\left(1+K^{-1}+\lambda_{1} K^{-1}\right),
$$

for a uniform $C_{2}$. The required upper bound for $\lambda_{1} / K$ follows from this.

Case 2. We now assume that $\delta \lambda_{1}<-\lambda_{n}$, with all the constants $D_{1}, D_{2}$ and $\delta$ fixed as in the previous case. We first use that $F^{n n} \geqslant \mathcal{F} / n$, as well as $\lambda_{n}^{2}>\delta^{2} \lambda_{1}^{2}$, to bound

$$
\begin{aligned}
\sum_{p} \frac{F^{k k}}{6 K}\left(\left|u_{p k}\right|^{2}+\left|u_{p \bar{k}}\right|^{2}\right) & \geqslant \frac{F^{n n}}{6 K}\left|u_{n \bar{n}}\right|^{2} \geqslant \frac{\mathcal{F}}{6 n K}\left|\lambda_{n}-\chi_{n \bar{n}}-W_{n \bar{n}}\right|^{2} \\
& \geqslant \frac{\mathcal{F}}{10 n K}\left|\lambda_{n}\right|^{2}-\frac{C \mathcal{F}}{K}(1+K) \geqslant \frac{\delta^{2}}{10 n K} \mathcal{F} \lambda_{1}^{2}-C \mathcal{F} .
\end{aligned}
$$

In (3.28) we now discard the positive first term and the term involving $\psi^{\prime \prime}$, and use this to obtain

$$
0 \geqslant-\frac{F^{k k}\left|\tilde{\lambda}_{1, k}\right|^{2}}{\lambda_{1}^{2}}+\frac{\delta^{2}}{10 n K} \mathcal{F} \lambda_{1}^{2}+\phi^{\prime \prime} F^{k k}\left|V_{k}\right|^{2}+\psi^{\prime} F^{k k} u_{k \bar{k}}-C\left(F^{k k} \lambda_{1}^{-1}\left|g_{1 \overline{1} k}\right|+\mathcal{F}\right) .
$$

To deal with the terms involving $F^{k k} u_{k \bar{k}}$ and $\left|g_{1 \overline{1} k}\right|$, we note that

$$
F^{k k}\left|u_{k \bar{k}}\right| \leqslant C \mathcal{F} \lambda_{1}
$$

and, since $g_{1 \overline{1} k}=\tilde{\lambda}_{1, k}+O\left(\lambda_{1}\right)$,

$$
C F^{k k} \lambda_{1}^{-1}\left|g_{1 \overline{1} k}\right| \leqslant C F^{k k} \lambda_{1}^{-1}\left|\tilde{\lambda}_{1, k}\right|+C \mathcal{F} \leqslant \frac{1}{2} \frac{F^{k k}\left|\tilde{\lambda}_{1, k}\right|^{2}}{\lambda_{1}^{2}}+C \mathcal{F} .
$$

Then, we obtain

$$
0 \geqslant-\frac{3}{2} \frac{F^{k k}\left|\tilde{\lambda}_{1, k}\right|^{2}}{\lambda_{1}^{2}}+\frac{\delta^{2}}{10 n K} \mathcal{F} \lambda_{1}^{2}+F^{k k} \phi^{\prime \prime}\left|V_{k}\right|^{2}-C \mathcal{F} \lambda_{1} .
$$

Using $\widetilde{H}_{k}=0$ we have, since $\psi^{\prime}$ is fixed now and bounded,

$$
\begin{aligned}
\frac{3}{2} \frac{F^{k k}\left|\tilde{\lambda}_{1, k}\right|^{2}}{\lambda_{1}^{2}}=\frac{3}{2} F^{k k}\left|\phi^{\prime} V_{k}+\psi^{\prime} u_{k}\right|^{2} & \leqslant 2 F^{k k}\left(\phi^{\prime}\right)^{2}\left|V_{k}\right|^{2}+C F^{k k}\left(\psi^{\prime}\right)^{2}\left|u_{k}\right|^{2} \\
& \leqslant F^{k k} \phi^{\prime \prime}\left|V_{k}\right|^{2}+C \mathcal{F} K .
\end{aligned}
$$


Returning to (3.56), we obtain, since we may assume $\lambda_{1} \geqslant K$,

$$
0 \geqslant \frac{\delta^{2} \lambda_{1}^{2}}{10 n K} \mathcal{F}-C \lambda_{1} \mathcal{F}
$$

Dividing by $\lambda_{1} \mathcal{F}$ gives the required bound for $\lambda_{1} / K$.

Then, we immediately deduce the bound (3.1), namely

$$
\sup _{M}|\sqrt{-1} \partial \bar{\partial} u|_{\alpha} \leqslant C\left(\sup _{M}|\nabla u|_{\alpha}^{2}+1\right)
$$

A blow-up argument as in $[34, \S 6]$ combined with a Liouville theorem $[34, \S 5]$ (see also [8], [39], [40]), shows that $\sup _{M}|\nabla u|_{\alpha}^{2} \leqslant C$, and so we get a uniform bound $|\Delta u| \leqslant C$. Here we remark that in the blow-up argument the only difference from the setup here (compared to [34]) is the presence of the term $W_{i \bar{j}}$. However, this term is linear in $\nabla u$ and so converges to zero uniformly on compact sets under the rescaling procedure of [34] (compare $[40, \S 6])$.

We can then apply the Evans-Krylov-type result in [36, Theorem 1.1] and deduce a uniform bound

$$
\|u\|_{C^{2, \beta}(M, \alpha)} \leqslant C
$$

for a uniform $0<\beta<1$. Differentiating the equation and applying a standard bootstrapping argument, we finally obtain uniform higher-order estimates.

\section{Proof of Theorem 1.4}

In this section, we explain how Theorem 1.4 follows from Theorem 2.2.

Write $*$ for the Hodge star operator with respect to $\alpha$. This acts on real $(n-1, n-1)$ forms as follows. Consider a real $(n-1, n-1)$-form $\Theta$ given by

$$
\Theta=(\sqrt{-1})^{n-1} \sum_{i, j} \operatorname{sgn}(i, j) \Theta_{i \bar{j}} d z^{1} \wedge d \bar{z}^{1} \wedge \ldots \wedge \widehat{d z^{i}} \wedge d \bar{z}^{i} \wedge \ldots \wedge d z^{j} \wedge \widehat{d \bar{z}} \bar{z}^{j} \wedge \ldots \wedge d z^{n} \wedge d \bar{z}^{n}
$$

with

$$
\operatorname{sgn}(i, j)= \begin{cases}1, & \text { if } i \leqslant j \\ -1, & \text { if } i>j\end{cases}
$$

If we are computing at a point in coordinates so that $\alpha_{i j}=\delta_{i j}$, then

$$
* \Theta=\sqrt{-1} \sum_{i, j} \Theta_{i \bar{j}} d z^{j} \wedge d \bar{z}^{i}
$$


A basic property is that for any Hermitian metric $\omega$ we have (see [39, §2], for example)

$$
\left(\frac{\omega^{n}}{\alpha^{n}}\right)^{n-1}=\frac{\left(*\left(\omega^{n-1}\right)\right)^{n}}{\left(*\left(\alpha^{n-1}\right)\right)^{n}}=\frac{\left(*\left(\omega^{n-1}\right)\right)^{n}}{((n-1) ! \alpha)^{n}} .
$$

Then, taking $\omega$ as in Theorem 1.4, we see that equation (1.7) is equivalent to

$$
\log \frac{\left(*\left(\omega^{n-1}\right)\right)^{n}}{((n-1) ! \alpha)^{n}}=h
$$

with $h=(n-1)(F+b)$ being a smooth function. Recall that

$$
\omega^{n-1}=\alpha_{0}^{n-1}+\sqrt{-1} \partial \bar{\partial} u \wedge \alpha^{n-2}+\operatorname{Re}\left(\sqrt{-1} \partial u \wedge \bar{\partial}\left(\alpha^{n-2}\right)\right) .
$$

As in [39], we have

$$
\frac{1}{(n-1) !} *\left(\sqrt{-1} \partial \bar{\partial} u \wedge \alpha^{n-2}\right)=\frac{1}{n-1}((\Delta u) \alpha-\sqrt{-1} \partial \bar{\partial} u) .
$$

Define

$$
Z_{i \bar{j}}=\left(\frac{1}{(n-1) !} * \operatorname{Re}\left(\sqrt{-1} \partial u \wedge \bar{\partial}\left(\alpha^{n-2}\right)\right)\right)_{i \bar{j}}
$$

A straightforward but long calculation gives

$$
\begin{gathered}
Z_{i \bar{j}}=\frac{1}{2(n-1)}\left(\alpha^{p \bar{q}} \alpha^{k \bar{\ell}} u_{p} \bar{T}_{q \ell \bar{k}} \alpha_{i \bar{j}}-\alpha^{k \bar{\ell}} u_{i} \bar{T}_{j \ell \bar{k}}-\alpha^{k \bar{\ell}} u_{k} \bar{T}_{\ell j \bar{i}}\right. \\
\left.+\alpha^{p \bar{q}} \alpha^{k \bar{\ell}} u_{\bar{q}} T_{p k \bar{\ell}} \alpha_{i \bar{j}}-\alpha^{k \bar{\ell}} u_{j} T_{i k \bar{\ell}}-\alpha^{k \bar{\ell}} u_{\bar{\ell}} T_{k i \bar{j}}\right)
\end{gathered}
$$

where we are writing $T_{i j}^{k}$ for the torsion of $\alpha$, and $T_{i j \bar{\ell}}=T_{i j}^{k} \alpha_{k \ell}$. An important point to note is that, since the torsion is skew-symmetric $\left(T_{i j \bar{\ell}}=-T_{j i \bar{\ell}}\right)$, in orthonormal coordinates for $\alpha$ we see that $Z_{i \bar{j}}$ is independent of $u_{\bar{i}}$ and $u_{j}$, and that $\nabla_{i} Z_{i \bar{i}}$ is independent of $u_{\bar{i}}$. Indeed, in local orthonormal coordinates for $\alpha$, we have

$$
Z_{i \bar{i}}=\frac{1}{2(n-1)}\left(\sum_{p \neq i} \sum_{k \neq i} u_{p} \bar{T}_{p k \bar{k}}+\sum_{p \neq i} \sum_{k \neq i} u_{\bar{p}} T_{p k \bar{k}}\right)
$$

and, for $i \neq j$,

$$
Z_{i \bar{j}}=\frac{1}{2(n-1)}\left(-\sum_{k \neq j}\left(u_{i} \bar{T}_{j k \bar{k}}+u_{k} \bar{T}_{k j \bar{i}}\right)-\sum_{k \neq i}\left(u_{\bar{j}} T_{i k \bar{k}}+u_{\bar{k}} T_{k i \bar{j}}\right)\right),
$$

using the skew-symmetry of the torsion. Also,

$$
\nabla_{i} Z_{i \bar{i}}=\frac{1}{2(n-1)}\left(\sum_{p \neq i} \sum_{k \neq i}\left(u_{p} \nabla_{i} \bar{T}_{p k \bar{k}}+\nabla_{i} u_{p} \bar{T}_{p k \bar{k}}\right)+\sum_{p \neq i} \sum_{k \neq i}\left(u_{\bar{p}} \nabla_{i} T_{p k \bar{k}}+\nabla_{i} u_{\bar{p}} T_{p k \bar{k}}\right)\right)
$$


and the statement follows. We also define

$$
\tilde{\chi}_{i \bar{j}}=\left(\frac{1}{(n-1) !} *\left(\alpha_{0}^{n-1}\right)\right)_{i \bar{j}} .
$$

Given this, we see that (4.1) is equivalent to

$$
\log \left(\mu_{1} \ldots \mu_{n}\right)=h
$$

where $\mu_{i}$ are the eigenvalues of $\alpha^{i \bar{p}} \tilde{g}_{j \bar{p}}$, for $\tilde{g}$ given by

$$
\tilde{g}_{i \bar{j}}=\tilde{\chi}_{i \bar{j}}+\frac{1}{n-1}\left((\Delta u) \alpha_{i \bar{j}}-u_{i \bar{j}}\right)+Z_{i \bar{j}}
$$

Since $\tilde{\chi}_{i \bar{j}}$ is positive definite, we have that 0 is a $\mathcal{C}$-subsolution. From the discussion in $\S 2$, it is now immediate to see that this equation falls into the setup of Theorem 2.2 , and so we obtain the uniform a-priori estimate (3.57). Therefore, Theorem 1.4 follows from [40, Theorem 1.7].

\section{References}

[1] Angella, D., Cohomological Aspects in Complex Non-Kähler Geometry. Lecture Notes in Mathematics, 2095. Springer, Cham, 2014.

[2] Bєocki, Z., On uniform estimate in Calabi-Yau theorem. Sci. China Ser. A, 48 (2005), $244-247$.

[3] Caffarelli, L., Nirenberg, L. \& Spruck, J., The Dirichlet problem for nonlinear second-order elliptic equations. III. Functions of the eigenvalues of the Hessian. Acta Math., 155 (1985), 261-301.

[4] Cherrier, P., Équations de Monge-Ampère sur les variétés hermitiennes compactes. Bull. Sci. Math., 111 (1987), 343-385.

[5] Chou, K.-S. \& Wang, X.-J., A variational theory of the Hessian equation. Comm. Pure Appl. Math., 54 (2001), 1029-1064.

[6] Chu, J., The complex Monge-Ampère equation on some compact Hermitian manifolds. Pacific J. Math., 276 (2015), 369-386.

[7] Collins, T.C. \& SzÉkelyhidi, G., Convergence of the $J$-flow on toric manifolds. J. Differential Geom., 107 (2017), 47-81.

[8] Dinew, S. \& KoŁodziej, S., Liouville and Calabi-Yau type theorems for complex Hessian equations. Amer. J. Math., 139 (2017), 403-415.

[9] Fino, A., Grantcharov, G. \& Vezzoni, L., Astheno-Kähler and balanced structures on fibrations. To appear in Int. Math. Res. Not.

[10] Fu, J., Wang, Z. \& Wu, D., Form-type Calabi-Yau equations. Math. Res. Lett., 17 (2010), 887-903.

[11] — Form-type equations on Kähler manifolds of nonnegative orthogonal bisectional curvature. Calc. Var. Partial Differential Equations, 52 (2015), 327-344. 
[12] Gauduchon, P., Le théorème de l'excentricité nulle. C. R. Acad. Sci. Paris Sér. A-B, 285 (1977), A387-A390.

[13] - La 1-forme de torsion d'une variété hermitienne compacte. Math. Ann., 267 (1984), 495-518.

[14] GuAn, B., Second-order estimates and regularity for fully nonlinear elliptic equations on Riemannian manifolds. Duke Math. J., 163 (2014), 1491-1524.

[15] — The Dirichlet problem for fully nonlinear elliptic equations on Riemannian manifolds. Preprint, 2014. arXiv:1403.2133 [math.AP].

[16] Guan, B. \& JiaO, H., Second order estimates for Hessian type fully nonlinear elliptic equations on Riemannian manifolds. Calc. Var. Partial Differential Equations, 54 (2015), 2693-2712.

[17] Guan, B. \& Li, Q., Complex Monge-Ampère equations and totally real submanifolds. Adv. Math., 225 (2010), 1185-1223.

[18] Harvey, F. R. \& Lawson, H. B., JR., Dirichlet duality and the nonlinear Dirichlet problem on Riemannian manifolds. J. Differential Geom., 88 (2011), 395-482.

[19] - Geometric plurisubharmonicity and convexity: an introduction. Adv. Math., 230 (2012), $2428-2456$

[20] Hou, Z., MA, X.-N. \& Wu, D., A second order estimate for complex Hessian equations on a compact Kähler manifold. Math. Res. Lett., 17 (2010), 547-561.

[21] Jost, J. \& YAU, S.-T., A nonlinear elliptic system for maps from Hermitian to Riemannian manifolds and rigidity theorems in Hermitian geometry. Acta Math., 170 (1993), 221254. Correction in Acta Math., 173 (1994), 307.

[22] Latorre, A. \& UGarte, L., On non-Kähler compact complex manifolds with balanced and astheno-Kähler metrics. C. R. Math. Acad. Sci. Paris, 355 (2017), 90-93.

[23] LI, Y., A priori estimates for Donaldson's equation over compact Hermitian manifolds. Calc. Var. Partial Differential Equations, 50 (2014), 867-882.

[24] LI, Y. Y., Some existence results for fully nonlinear elliptic equations of Monge-Ampère type. Comm. Pure Appl. Math., 43 (1990), 233-271.

[25] LI, S.-Y., On the Dirichlet problems for symmetric function equations of the eigenvalues of the complex Hessian. Asian J. Math., 8 (2004), 87-106.

[26] Michelsohn, M. L., On the existence of special metrics in complex geometry. Acta Math., 149 (1982), 261-295.

[27] NiE, X., Regularity of a complex Monge-Ampère equation on Hermitian manifolds. Comm. Anal. Geom., 22 (2014), 833-856.

[28] Phong, D. H., Song, J. \& Sturm, J., Complex Monge-Ampère equations, in Surveys in Differential Geometry. Vol. XVII, Surv. Differ. Geom., 17, pp. 327-410. Int. Press, Boston, MA, 2012.

[29] Popovici, D., Deformation limits of projective manifolds: Hodge numbers and strongly Gauduchon metrics. Invent. Math., 194 (2013), 515-534.

[30] - Aeppli cohomology classes associated with Gauduchon metrics on compact complex manifolds. Bull. Soc. Math. France, 143 (2015), 763-800.

[31] Spruck, J., Geometric aspects of the theory of fully nonlinear elliptic equations, in Global Theory of Minimal Surfaces, Clay Math. Proc., 2, pp. 283-309. Amer. Math. Soc., Providence, RI, 2005.

[32] Sun, W., On a class of fully nonlinear elliptic equations on closed Hermitian manifolds. J. Geom. Anal., 26 (2016), 2459-2473.

[33] — On a class of fully nonlinear elliptic equations on closed Hermitian manifolds II: $L^{\infty}$ estimate. Comm. Pure Appl. Math., 70 (2017), 172-199. 
[34] SzÉKelyhidi, G., Fully non-linear elliptic equations on compact Hermitian manifolds. To appear in J. Differential Geom.

[35] Tosatti, V., Non-Kähler Calabi-Yau manifolds, in Analysis, Complex Geometry, and Mathematical Physics, Contemp. Math., 644, pp. 261-277. Amer. Math. Soc., Providence, RI, 2015.

[36] Tosatti, V., Wang, Y., Weinkove, B. \& Yang, X., $C^{2, \alpha}$ estimates for nonlinear elliptic equations in complex and almost complex geometry. Calc. Var. Partial Differential Equations, 54 (2015), 431-453.

[37] Tosatti, V. \& Weinkove, B., The complex Monge-Ampère equation on compact Hermitian manifolds. J. Amer. Math. Soc., 23 (2010), 1187-1195.

[38] - Estimates for the complex Monge-Ampère equation on Hermitian and balanced manifolds. Asian J. Math., 14 (2010), 19-40.

[39] - The Monge-Ampère equation for $(n-1)$-plurisubharmonic functions on a compact Kähler manifold. J. Amer. Math. Soc., 30 (2017), 311-346.

[40] - Hermitian metrics, $(n-1, n-1)$ forms and Monge-Ampère equations. To appear in $J$. Reine Angew. Math.

[41] Trudinger, N. S., On the Dirichlet problem for Hessian equations. Acta Math., 175 (1995), $151-164$.

[42] Urbas, J., Hessian equations on compact Riemannian manifolds, in Nonlinear Problems in Mathematical Physics and Related Topics, II, Int. Math. Ser. (N. Y.), 2, pp. 367-377. Kluwer/Plenum, New York, 2002.

[43] YAu, S.-T., On the Ricci curvature of a compact Kähler manifold and the complex MongeAmpère equation. I. Comm. Pure Appl. Math., 31 (1978), 339-411.

[44] Zhang, D., Hessian equations on closed Hermitian manifolds. Pacific J. Math., 291 (2017), 485-510.

[45] Zhang, X., A priori estimates for complex Monge-Ampère equation on Hermitian manifolds. Int. Math. Res. Not., 19 (2010), 3814-3836.

[46] ZhANG, X. \& Zhang, X., Regularity estimates of solutions to complex Monge-Ampère equations on Hermitian manifolds. J. Funct. Anal., 260 (2011), 2004-2026.

GÁBor SzÉKELYHIDI

Department of Mathematics

University of Notre Dame

255 Hurley

Notre Dame, IN 46556

U.S.A.

gszekely@nd.edu

\section{Ben WeINKOVE}

Department of Mathematics

Northwestern University

2033 Sheridan Road

Evanston, IL 60208

U.S.A.

weinkove@math.northwestern.edu

\author{
VAlentino Tosatti \\ Department of Mathematics \\ Northwestern University \\ 2033 Sheridan Road \\ Evanston, IL 60208 \\ U.S.A. \\ tosatti@math.northwestern.edu
}

Received October 12, 2015 